INTER NATIONAL MONETARY FUND

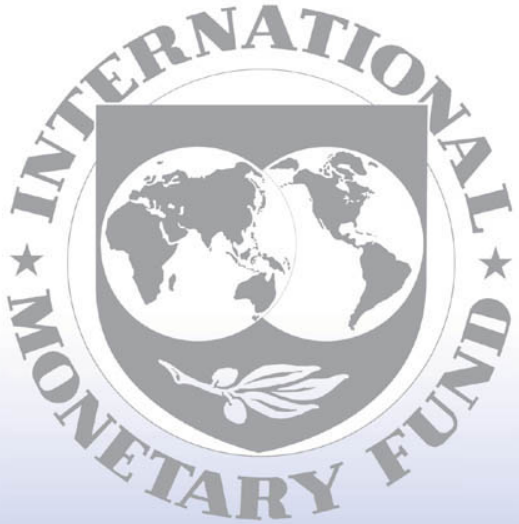

Staff

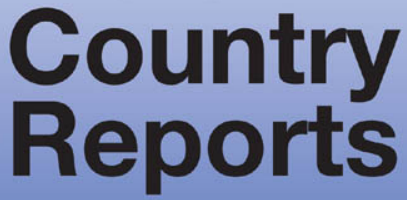




\section{Kuwait: Statistical Appendix}

This Statistical Appendix for Kuwait was prepared by a staff team of the International Monetary Fund as background documentation for the periodic consultation with the member country. It is based on the information available at the time it was completed on January 16, 2004. The views expressed in this document are those of the staff team and do not necessarily reflect the views of the government of Kuwait or the Executive Board of the IMF.

The policy of publication of staff reports and other documents by the IMF allows for the deletion of market-sensitive information.

To assist the IMF in evaluating the publication policy, reader comments are invited and may be sent by e-mail to publicationpolicy@imf.org.

Copies of this report are available to the public from

International Monetary Fund $\bullet$ Publication Services

700 19th Street, N.W. • Washington, D.C. 20431

Telephone: (202) 6237430 • Telefax: (202) 6237201

E-mail: publications@imf.org • Internet: http://www.imf.org

Price: $\$ 15.00$ a copy

\section{International Monetary Fund \\ Washington, D.C.}


This page intentionally left blank 


\section{INTERNATIONAL MONETARY FUND}

\section{KUWAIT}

\section{Statistical Appendix}

Prepared by a staff team consisting of Zubair Iqbal, Mohamed Elhage, Van Can Thai, Bright Okogu, and Hajime Takizawa (all MCD)

Approved by the Middle East and Central Asia Department

January 16, 2004

Contents

Basic Data, 1998-2003

Tables

1. Sectoral Origin of Gross Domestic Product at Current Prices 1997-2002 ……........... $\underline{6}$

2. Sectoral Origins of Gross Domestic Product at Constant 1995 Prices, 1997-2002 … 7

3. Gross Domestic Expenditure at Current Market Prices, 1997-2002 ……….............. $\frac{8}{9}$

4. Gross Domestic Expenditure at Constant 1995 Prices, 1997-2002 …......................... 9

5. Production and Disposal of Crude Oil and LPG, 1997-2003 ….............................10

6. Consumer Prices and Index, 1997-2003 ………............................................

7. Whole Sale Price Index, 1997-2003 ………................................................

8. Output of Major Industrial Products, 1997-2002 ……...................................... $\frac{13}{14}$

9. Agricultural and Fisheries Production, 1996/97-2001/02 ……………………....... 14

10. Population and Employment, 1997-2002 ………............................................ $\frac{15}{16}$

11. Distribution of Employees by Economic Activity and Nationality, 1999-2002 _....... $\frac{16}{16}$

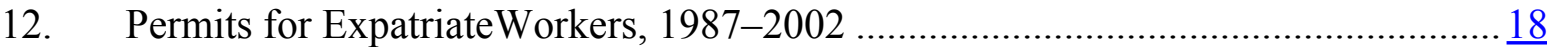

13. Number of Construction Permits Issued, 1988-2001 ………................................

14. Summary of Government Finance, 1998/99-2003/04 …………………………....

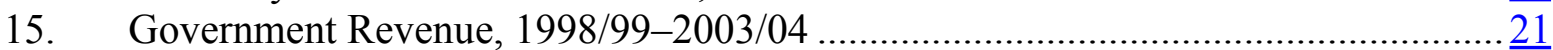

16. Government Current Expenditure, 1998/99-2003/04..........................................22

17. Government Capital Expenditures and Land Purchases, 1998/99-2003/04 …….......23

18. Government Domestic Subsidies and Transfers, 1998/99-2003/04 ………..............24

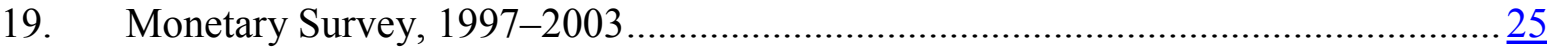

20. Monetary Accounts for the Central Bank, 1997-2003 …......................................27

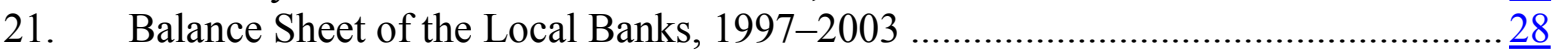

22. Distribution of Local Bank Credit Outstanding to the Private Sector, 1997-2003 ...229

23. Structure of Interest Rates, 1997-2003 ………..............................................

24. Interest Rates on Kuwaiti Dinar and U.S. Dollar Deposits with Local Banks, 1997-2003 
25. Balance Sheet of the Investment Companies, 1997-2003

26. Debt Collection Program-Repayments in 1995-2001 Repayment by Debtors

Under the Law 41/93 $\underline{33}$

27. Summary of the Balance of Payments, 1997-2002 …………………….................

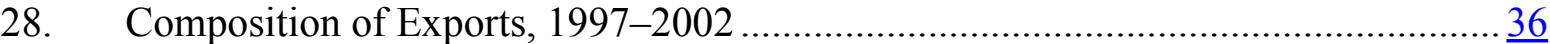

29. Composition of Imports, 1997-2002 ………….............................................

30. External Services, Investment Income, and Current Transfers, 1997-2002 ...............

31. Capital and Financial Account, 1998-2002 ....................................................... 39

32. Reserves and Net Foreign Assets, 1997-2002 …...........................................

33. Aggregate Banking Soundness Indicators, 1997-2003 ………………………....... $\frac{42}{44}$

34. Selected Stock Market Indicators, 1997-2003 ………………………...................44

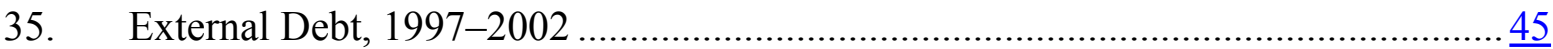


Kuwait: Basic Data

(Quota: SDR 1,381.10 million)

I. Social and Demographic Indicators, 2001

Area (thousand sq. km.)

17,820

Population

Total (in millions)

2.31

Annual rate of growth (in percent)

4.05

Density (estimated numbers of inhabitants per sq. km.)

GDP per capita (US\$)

Population characteristics

Life expectancy at birth (in years)

Overall

76.74

Female

78.84

Infant mortality (per 1000 live births)

Child death rate, under 5 years (per 1000 live births)

Education

Illiteracy rate (percent of population aged 15 and over)

17.56

Male (percent of population aged 15 and over)

15.70

Female (percent of population aged 15 and over)

19.71 
Kuwait: Basic Data

II. Selected Economic Indicators, 1998-2003

\begin{tabular}{|c|c|c|c|c|c|c|}
\hline & 1998 & 1999 & 2000 & 2001 & 2002 & $\begin{array}{r}\text { Est. } \\
2003\end{array}$ \\
\hline Nominal GDP (market prices, US\$ millions) & 25,945 & 30,077 & 37,017 & 34,236 & 35,333 & 42,372 \\
\hline Crude oil production (millions barrels per day) & 2.05 & 1.87 & 1.98 & 1.95 & 1.75 & 2.03 \\
\hline Kuwait crude export price (US\$ per barrel) & 10.3 & 16.1 & 25.0 & 21.2 & 22.9 & 25.6 \\
\hline & \multicolumn{6}{|c|}{ (Percent changes) } \\
\hline \multicolumn{7}{|l|}{ Production and prices } \\
\hline Nominal GDP & -14.1 & 16.0 & 23.8 & -7.6 & 2.3 & 18.2 \\
\hline Nominal non-oil GDP & 5.9 & 3.9 & 3.5 & 4.2 & 6.6 & 7.1 \\
\hline Real GDP & 3.6 & -1.7 & 1.9 & 0.6 & -0.4 & 9.9 \\
\hline Real oil GDP & 1.8 & -6.5 & 2.3 & -3.2 & -8.0 & 15.5 \\
\hline Real non-oil GDP & 5.2 & 2.2 & 1.8 & 3.4 & 5.0 & 6.5 \\
\hline \multirow[t]{2}{*}{ Consumer price index } & 0.1 & 3.0 & 1.8 & 1.7 & 1.4 & 2.0 \\
\hline & \multicolumn{6}{|c|}{ (Changes in percent of beginning broad money stock) } \\
\hline \multicolumn{7}{|l|}{ Money and credit } \\
\hline Foreign assets (net) & -1.9 & 1.5 & 11.4 & 6.5 & -4.1 & -3.4 \\
\hline Domestic assets (net) & 1.1 & 0.1 & -5.1 & 6.3 & 8.8 & 16.0 \\
\hline Claims on government (net) & -1.4 & -2.7 & -7.3 & -4.1 & 0.2 & -3.5 \\
\hline Claims on nongovernment sector & 7.3 & 3.6 & 3.9 & 12.0 & 10.6 & 18.5 \\
\hline \multirow[t]{2}{*}{ Broad money } & -0.8 & 1.6 & 6.3 & 12.8 & 4.8 & 12.5 \\
\hline & \multicolumn{6}{|c|}{ (Percent per year) } \\
\hline \multicolumn{7}{|l|}{ Interest rates $1 /$} \\
\hline Kuwaiti dinar three-month deposits & 5.9 & 5.3 & 5.4 & 3.7 & 2.2 & 1.5 \\
\hline U.S. dollar three-month deposits & 5.1 & 4.9 & 6.0 & 3.3 & 1.3 & 0.7 \\
\hline & \multicolumn{6}{|c|}{ (In millions of U.S. dollars unless otherwise indicated) } \\
\hline \multicolumn{7}{|l|}{ External sector } \\
\hline Exports & 9,616 & 12,225 & 19,476 & 16,246 & 15,365 & 20,992 \\
\hline Of which: oil and refined products & 8,470 & 11,027 & 18,184 & 14,976 & 14,058 & 18,784 \\
\hline Imports & $-7,715$ & $-6,708$ & $-6,451$ & $-7,049$ & $-8,118$ & $-10,241$ \\
\hline Current account & 2,215 & 5,013 & 14,672 & 8,328 & 4,190 & 8,046 \\
\hline In percent of GDP & 8.5 & 16.6 & 39.6 & 24.3 & 11.9 & 19.0 \\
\hline Overall balance & 288 & 894 & 2,241 & 2,827 & -741 & $-1,907$ \\
\hline In percent of GDP & 1.1 & 3.0 & 6.1 & 8.3 & -2.1 & -4.5 \\
\hline International reserve assets & 4,034 & 4,928 & 7,170 & 9,997 & 9,256 & 7,348 \\
\hline In months of imports of goods and services & 3.7 & 5.0 & 7.4 & 9.7 & 8.3 & 5.6 \\
\hline Total external debt (incl private sector) & 9,938 & 10,057 & 9,955 & 11,095 & 12,939 & 12,480 \\
\hline \multirow[t]{2}{*}{ In percent of GDP } & 38.3 & 33.4 & 26.9 & 32.4 & 36.6 & 29.5 \\
\hline & \multicolumn{6}{|c|}{ (Percentage change) } \\
\hline \multicolumn{7}{|l|}{ Exchange rates } \\
\hline Exchange rates (US\$ per KD, period average) 2/ & 3.28 & 3.28 & 3.26 & 3.26 & 3.29 & 3.35 \\
\hline Nominal effective exchange rate $3 /$ & 5.5 & -0.6 & 4.7 & 5.8 & -0.7 & -6.4 \\
\hline
\end{tabular}


Real effective exchange rate $3 /$

Kuwait: Basic Data

Real effective exchange rate 3/

$3.1 \quad 0.9$

4.2

5.1

$-1.5$

$-6.8$

II. Selected Economic Indicators, 1998-2003 (concluded)

\begin{tabular}{|c|c|c|c|c|c|c|}
\hline & \multicolumn{2}{|c|}{$1998 / 991999 / 2000$} & \multirow[t]{2}{*}{$2000 / 01$} & \multirow[t]{2}{*}{$2001 / 02$} & \multirow[t]{2}{*}{$2002 / 03$} & \multirow[t]{2}{*}{$\frac{\text { Est. }}{2003 / 04}$} \\
\hline & & & & & & \\
\hline \multicolumn{7}{|l|}{ Public finance 4/ } \\
\hline Total revenue, of which: & 46.4 & 68.7 & 77.8 & 66.3 & 68.6 & 61.3 \\
\hline Oil and gas revenue & 26.4 & 46.7 & 55.3 & 42.9 & 49.0 & 42.3 \\
\hline Investment income & 16.6 & 19.3 & 18.2 & 16.6 & 14.4 & 14.2 \\
\hline Total expenditures & 48.7 & 39.5 & 39.2 & 44.8 & 43.2 & 42.6 \\
\hline Current & 43.1 & 36.0 & 36.0 & 40.7 & 38.2 & 37.6 \\
\hline Capital & 5.6 & 3.5 & 3.1 & 4.1 & 5.0 & 5.0 \\
\hline Fiscal balance (deficit -) & -2.3 & 29.3 & 38.7 & 21.5 & 24.6 & 18.6 \\
\hline Fiscal balance excluding investment income (defiı & -18.9 & 10.0 & 20.5 & 4.9 & 10.2 & 4.4 \\
\hline
\end{tabular}

Sources: Data provided by the authorities; and Fund staff estimates.

1/ In 2003, up to September.

2/ For 2003, average for January-October.

$3 /$ In 2003, up to October.

4/ The fiscal numbers are for fiscal year which was changed from July-June to April-March effective 2001/02. 
Table 1. Kuwait: Sectoral Origin of Gross Domestic Product at Current Prices, 1997-2002

(In millions of Kuwaiti dinars)

\begin{tabular}{|c|c|c|c|c|c|c|}
\hline & 1997 & 1998 & 1999 & 2000 & 2001 & $\frac{\text { Prel. }}{2002}$ \\
\hline Oil sector (crude oil, gas, and refining) & 4,539 & 2,965 & 4,036 & 6,046 & 4,961 & 4,836 \\
\hline Non-oil sector & 4,886 & 5,243 & 5,496 & 5,710 & 5,940 & 6,307 \\
\hline Agriculture and fisheries & 36 & 35 & 39 & 42 & 48 & 48 \\
\hline Mining (non-oil) and quarrying & 1 & 1 & 1 & 1 & 1 & 1 \\
\hline Manufacturing & 311 & 316 & 308 & 300 & 292 & 306 \\
\hline Food, beverages, and tobacco & 55 & 64 & 65 & 69 & 61 & 62 \\
\hline Textiles, clothing, and leather products & 38 & 39 & 35 & 36 & 36 & 37 \\
\hline Wood and wood products & 15 & 15 & 15 & 13 & 13 & 14 \\
\hline Paper, printing, and publishing & 28 & 29 & 29 & 28 & 29 & 29 \\
\hline Chemicals, fertilizers, and plastics & 55 & 42 & 39 & 37 & 37 & 42 \\
\hline Nonmetallic minerals & 42 & 47 & 47 & 45 & 46 & 49 \\
\hline Basic metals & 4 & 4 & 5 & 5 & 5 & 5 \\
\hline Fabricated metal products & 68 & 71 & 68 & 60 & 60 & 62 \\
\hline Other manufacturing & 6 & 4 & 4 & 7 & 7 & 7 \\
\hline Electricity, gas, and water & 148 & 230 & 237 & 246 & 251 & 276 \\
\hline Construction & 235 & 231 & 240 & 249 & 255 & 259 \\
\hline Wholesale and retail trade & 557 & 587 & 609 & 593 & 629 & 679 \\
\hline Hotels and restaurants & 74 & 81 & 83 & 84 & 84 & 85 \\
\hline Transport, storage, and communications & 399 & 433 & 487 & 506 & 552 & 564 \\
\hline Financial institutions and insurance & 400 & 503 & 573 & 617 & 693 & 704 \\
\hline Real estate and business services & 687 & 652 & 672 & 710 & 713 & 752 \\
\hline Community, social, and personal services & 2,037 & 2,174 & 2,248 & 2,364 & 2,422 & 2,633 \\
\hline Public administration and defense & 926 & 959 & 993 & 1,008 & 1,008 & 1,108 \\
\hline Personal and household services & 177 & 232 & 233 & 263 & 278 & 289 \\
\hline Other & 935 & 982 & 1,021 & 1,092 & 1,137 & 1,236 \\
\hline Imputed bank service charges & -302 & -385 & -442 & -476 & -489 & -487 \\
\hline GDP at factor cost & 9,124 & 7,822 & 9,089 & 11,280 & 10,412 & 10,656 \\
\hline Import duties & 83 & 84 & 80 & 77 & 84 & 81 \\
\hline GDP at current market prices & 9,207 & 7,907 & 9,170 & 11,357 & 10,496 & 10,738 \\
\hline
\end{tabular}

Source: Ministry of Planning, Central Statistics Office. 
Table 2. Kuwait: Sectoral Origins of Gross Domestic Product at Constant 1995 Prices, 1997-2002

(In millions of Kuwaiti dinars)

\begin{tabular}{|c|c|c|c|c|c|c|}
\hline & 1997 & 1998 & 1999 & 2000 & 2001 & $\frac{\text { Prel. }}{2002}$ \\
\hline Oil sector (crude oil, gas, and refining) & 3,752 & 3,820 & 3,571 & 3,651 & 3,533 & 3,250 \\
\hline Non-oil sector & 4,764 & 5,011 & 5,121 & 5,211 & 5,390 & 5,659 \\
\hline Agriculture and fisheries & 35 & 33 & 36 & 37 & 43 & 41 \\
\hline Agriculture and livestock & 27 & 26 & 30 & 31 & 39 & 37 \\
\hline Fisheries & 8 & 7 & 6 & 6 & 5 & 4 \\
\hline Mining (non-oil) and quarrying & 1 & 1 & 1 & 1 & 1 & 0 \\
\hline Manufacturing & 315 & 321 & 308 & 302 & 289 & 301 \\
\hline Petroleum and refining & 615 & 632 & 645 & 539 & 488 & 521 \\
\hline Food, beverages, and tobacco & 52 & 60 & 59 & 62 & 56 & 59 \\
\hline Textile, clothing, and leather products & 39 & 41 & 35 & 35 & 32 & 29 \\
\hline Wood and wood products & 12 & 12 & 12 & 11 & 11 & 11 \\
\hline Paper, printing, and publishing & 31 & 34 & 33 & 32 & 33 & 34 \\
\hline Chemicals, fertilizers, and plastics & 56 & 42 & 40 & 39 & 39 & 44 \\
\hline Nonmetallic minerals & 44 & 48 & 49 & 50 & 49 & 46 \\
\hline Basic metals & 6 & 7 & 7 & 7 & 7 & 7 \\
\hline Fabricated metal products & 69 & 72 & 69 & 59 & 56 & 63 \\
\hline Other manufacturing & 5 & 4 & 4 & 7 & 7 & 7 \\
\hline Electricity, gas, and water & 147 & 227 & 250 & 285 & 316 & 353 \\
\hline Construction & 228 & 227 & 234 & 241 & 239 & 247 \\
\hline Trade, hotels, and restaurants & 602 & 626 & 642 & 614 & 634 & 672 \\
\hline Transport, storage, and communications & 432 & 487 & 557 & 569 & 616 & 620 \\
\hline Financial institutions and insurance & 309 & 321 & 332 & 349 & 394 & 419 \\
\hline Real estate and business services & 664 & 621 & 627 & 651 & 647 & 675 \\
\hline Community, social, and personal services & 2,032 & 2,148 & 2,134 & 2,164 & 2,212 & 2,331 \\
\hline Public administration and defense & 974 & 1,013 & 1,003 & 1,001 & 1,019 & 1,062 \\
\hline Personal and household services & 179 & 235 & 220 & 244 & 285 & 299 \\
\hline Other & 879 & 900 & 912 & 919 & 908 & 970 \\
\hline Imputed bank service charges & -230 & -246 & -252 & -261 & -272 & -284 \\
\hline GDP at factor cost & 8,285 & 8,585 & 8,439 & 8,601 & 8,651 & 8,624 \\
\hline Import duties & 80 & 83 & 80 & 76 & 82 & 76 \\
\hline GDP at constant prices & 8,365 & 8,668 & 8,519 & 8,677 & 8,732 & 8,700 \\
\hline
\end{tabular}

Source: Ministry of Planning, Central Statistical Office. 
Table 3. Kuwait: Gross Domestic Expenditure at Current Market Prices, 1997-2002

\begin{tabular}{|c|c|c|c|c|c|c|}
\hline & 1997 & 1998 & 1999 & 2000 & 2001 & $\frac{\text { Prel. }}{2002}$ \\
\hline & \multicolumn{6}{|c|}{ (In millions of Kuwaiti dinars) } \\
\hline Final consumption & 6,730 & 7,020 & 7,239 & 7,443 & 7,899 & 8,824 \\
\hline Government & 2,452 & 2,412 & 2,463 & 2,485 & 2,521 & 2,838 \\
\hline Private $1 /$ & 4,279 & 4,608 & 4,776 & 4,958 & 5,377 & 5,986 \\
\hline Gross domestic investment & 1,256 & 1,459 & 1,335 & 868 & 910 & 979 \\
\hline Government & 470 & 371 & 357 & 202 & 225 & 262 \\
\hline Private & 786 & 1,088 & 978 & 666 & 685 & 717 \\
\hline Net exports of goods and nonfactor services & 1,221 & -572 & 596 & 3,046 & 1,687 & 934 \\
\hline Exports of goods and services & 4,866 & 3,468 & 4,212 & 6,534 & 5,490 & 5,184 \\
\hline Imports of goods and services & 3,645 & 4,040 & 3,616 & 3,488 & 3,803 & 4,250 \\
\hline Gross domestic product & 9,207 & 7,907 & 9,170 & 11,357 & 10,496 & 10,738 \\
\hline Net factor income from abroad & 1,904 & 1,788 & 1,555 & 2,055 & 1,503 & 1,021 \\
\hline Gross national income & 11,111 & 9,695 & 10,725 & 13,412 & 11,999 & 11,759 \\
\hline Net transfers & -457 & -541 & -610 & -600 & -637 & -652 \\
\hline Gross national disposable income & 10,654 & 9,154 & 10,115 & 12,812 & 11,362 & 11,107 \\
\hline Gross saving & 3,924 & 2,134 & 2,876 & 5,369 & 3,463 & 2,282 \\
\hline $\mathrm{S}-\mathrm{I}=\mathrm{CAB}$ & 2,668 & 675 & 1,541 & 4,501 & 2,553 & 1,303 \\
\hline Current account & 2,410 & 675 & 1,541 & 4,501 & 2,624 & 2,625 \\
\hline & \multicolumn{6}{|c|}{ (In percent of GDP) } \\
\hline \multicolumn{7}{|l|}{ Memorandum items: } \\
\hline Final consumption & 73.1 & 88.8 & 78.9 & 65.5 & 75.3 & 82.2 \\
\hline Government & 26.6 & 30.5 & 26.9 & 21.9 & 24.0 & 26.4 \\
\hline Private $1 /$ & 46.5 & 58.3 & 52.1 & 43.7 & 51.2 & 55.8 \\
\hline Gross domestic investment & 13.6 & 18.5 & 14.6 & 7.6 & 8.7 & 9.1 \\
\hline Government & 5.1 & 4.7 & 3.9 & 1.8 & 2.1 & 2.4 \\
\hline Private & 8.5 & 13.8 & 10.7 & 5.9 & 6.5 & 6.7 \\
\hline Saving & 42.6 & 27.0 & 31.4 & 47.3 & 33.0 & 21.3 \\
\hline Current account & 26.2 & 8.5 & 16.8 & 39.6 & 25.0 & 24.4 \\
\hline
\end{tabular}

Source: Ministry of Planning, Central Statistical Office.

1/ Includes government-owned enterprises. 
Table 4. Kuwait: Gross Domestic Expenditure at Constant 1995 Prices, 1997-2002

(In millions of Kuwaiti dinars)

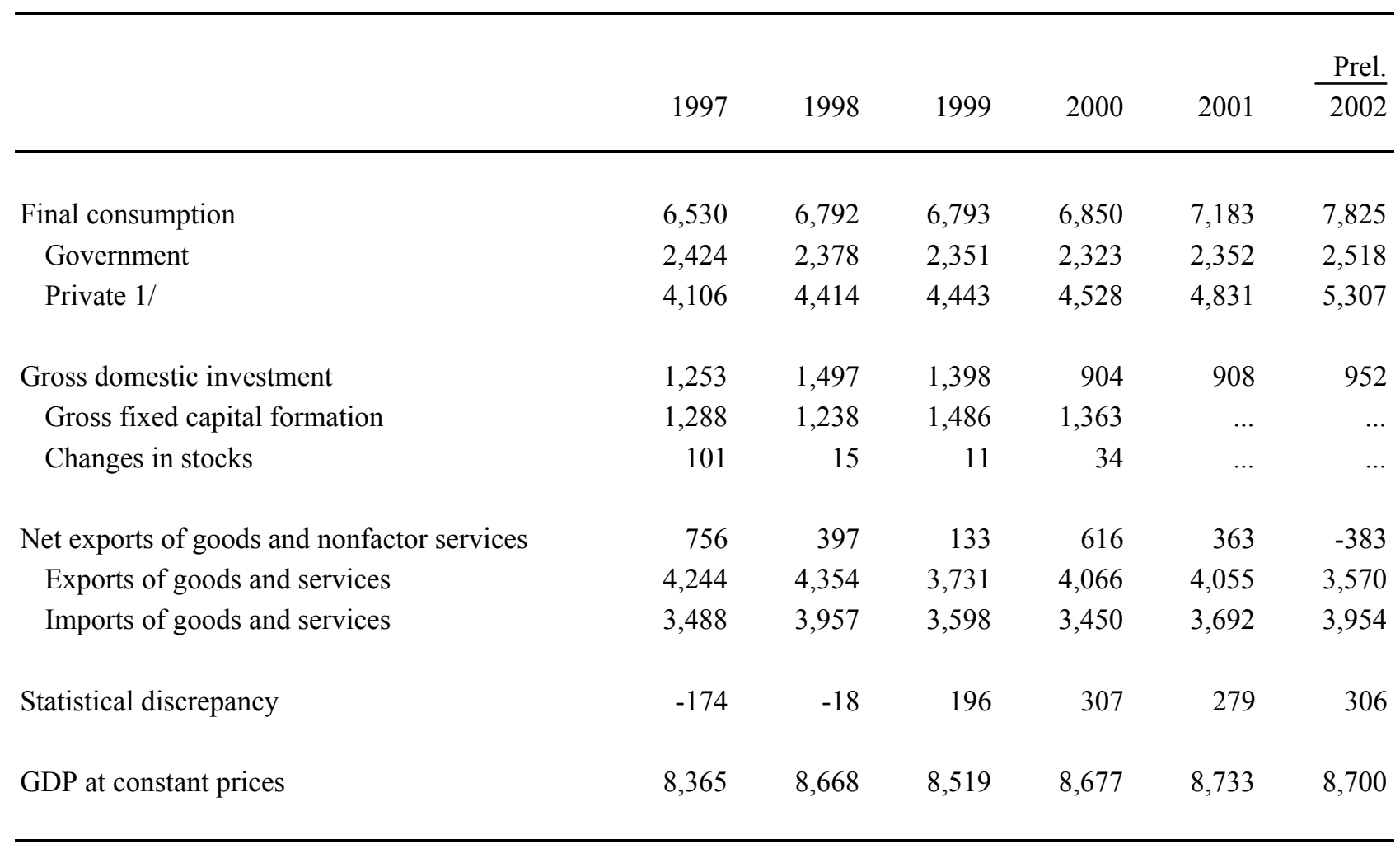

Sources: Ministry of Planning, Central Statistical Office; and Fund staff estimates.

1/ Includes government-owned enterprises. 
Table 5. Kuwait: Production and Disposal of Crude Oil and LPG, 1997-2003

(In thousands of barrels per day)

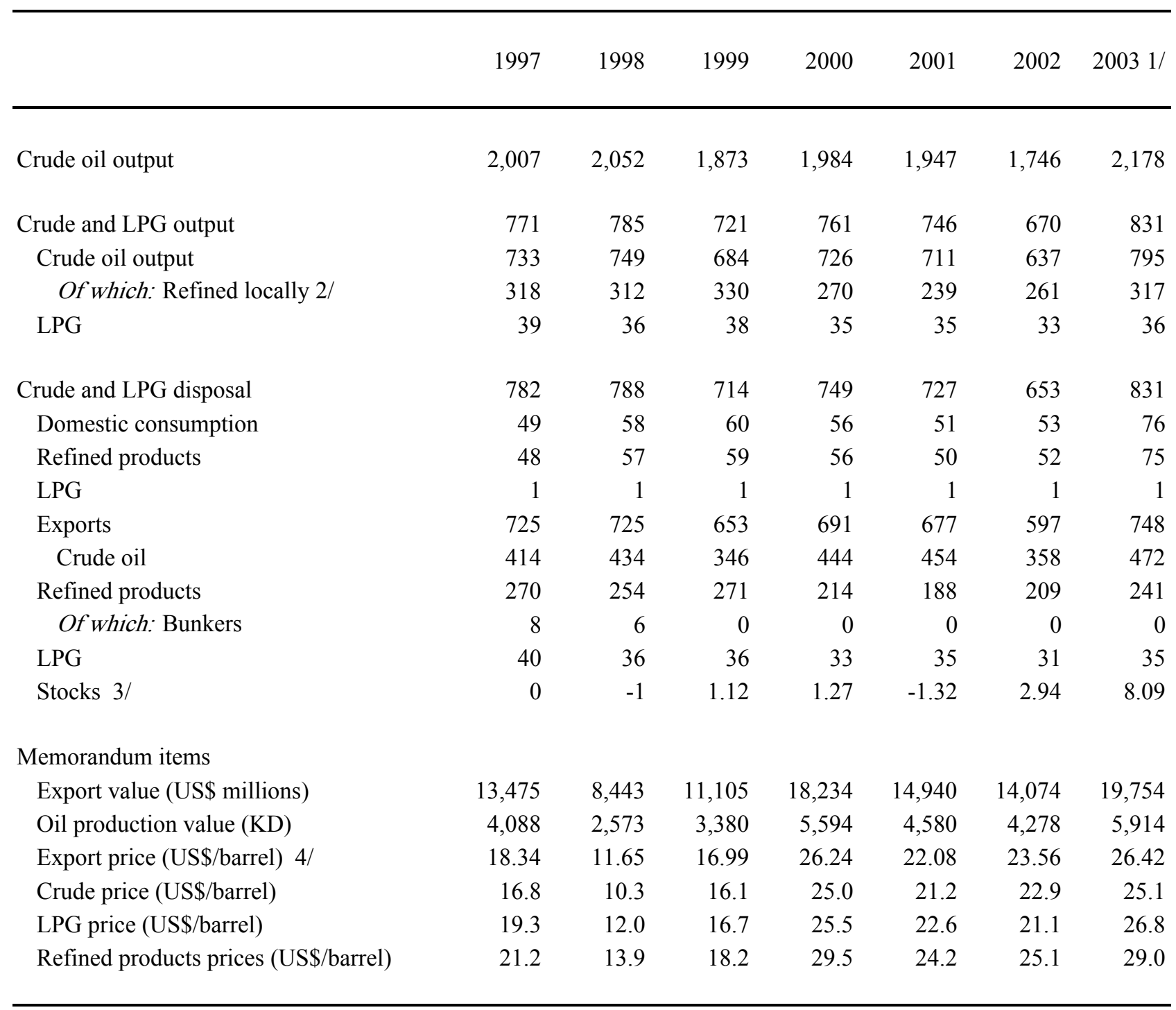

Sources: Kuwaiti authorities, oil industry journals, and Fund staff estimates.

1/ Data cover January-June 2003.

2/ Excludes bunkers and stocks.

3/ Includes statistical discrepancy.

4/ Average price of crude, LPG, and refined products. 
Table 6. Kuwait: Consumer Price Index, 1997-2003

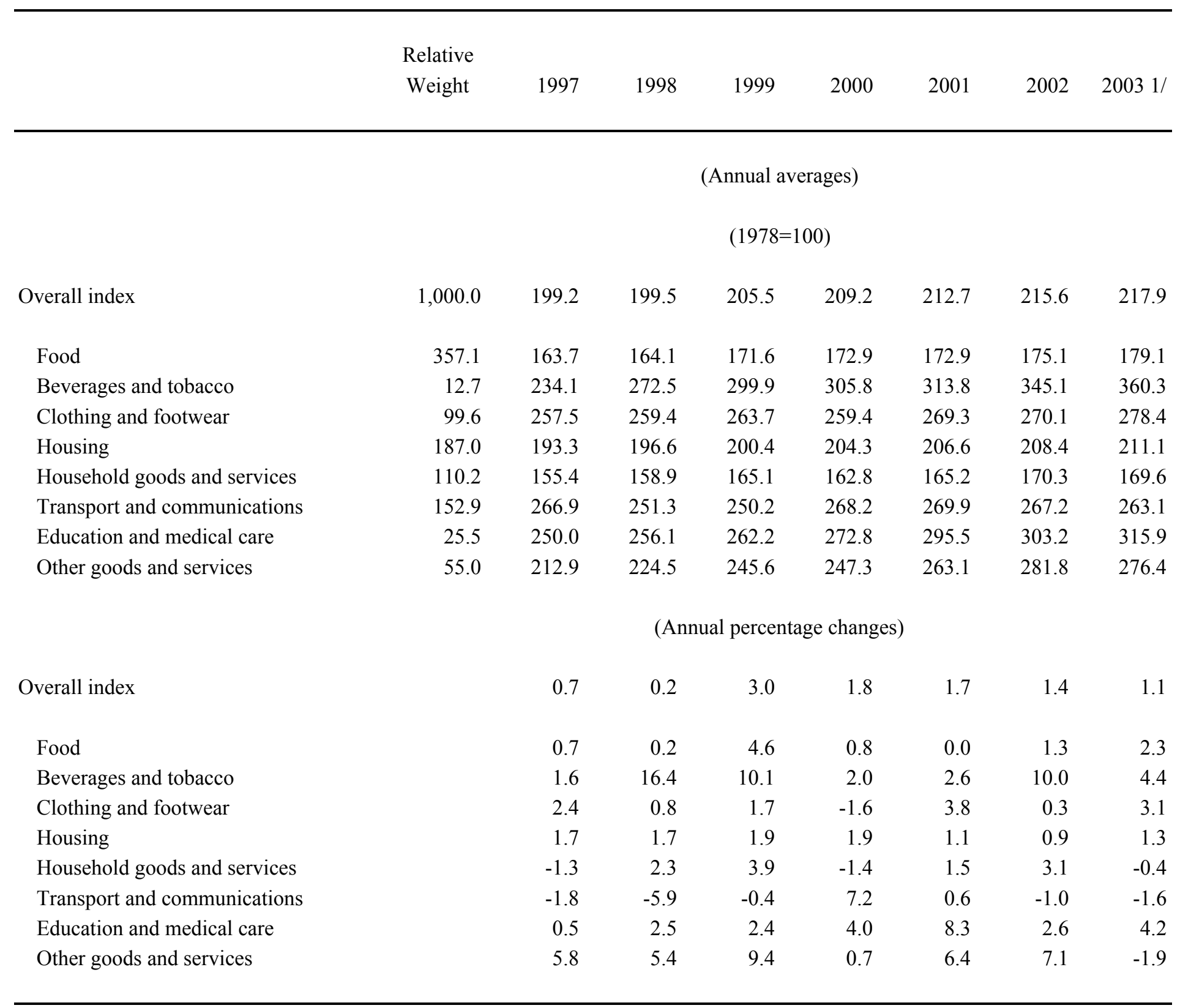

Source: Ministry of Planning, Central Statistical Office.

1/ Data cover January-July 2003. 
Table 7. Kuwait: Wholesale Price Index, 1997-2003

\begin{tabular}{|c|c|c|c|c|c|c|c|c|c|c|}
\hline & \multicolumn{3}{|c|}{ Relative Weights } & \multirow[b]{2}{*}{1997} & \multirow[b]{2}{*}{1998} & \multirow[b]{2}{*}{1999} & \multirow[b]{2}{*}{2000} & \multirow[b]{2}{*}{2001} & \multirow[b]{2}{*}{2002} & \multirow[b]{2}{*}{$20031 /$} \\
\hline & Imported & $\begin{array}{l}\text { Locally } \\
\text { Produced }\end{array}$ & $\begin{array}{c}\text { All } \\
\text { Items }\end{array}$ & & & & & & & \\
\hline All groups & 769.2 & 230.8 & $1,000.0$ & 167.1 & 164.4 & 162.4 & 163.1 & 166.3 & 171.8 & 174.7 \\
\hline Agriculture, livestock, and fishing & 47.4 & 6.1 & 53.4 & 121.2 & 124.3 & 121.5 & 124.8 & 120.0 & 127.0 & 128.1 \\
\hline Agriculture & 30.0 & 1.9 & 31.9 & 119.7 & 124.4 & 117.9 & 120.8 & 121.4 & 128.1 & 128.1 \\
\hline Livestock & 17.3 & 2.1 & 19.5 & 112.5 & 112.1 & 116.6 & 116.6 & 106.7 & 111.5 & 111.5 \\
\hline Fishing & 0.0 & 2.1 & 2.1 & 224.8 & 234.4 & 219.1 & 263.8 & 219.7 & 255.7 & 255.7 \\
\hline Mining (non-oil) and quarrying & 0.0 & 7.8 & 7.8 & 104.6 & 103.5 & 102.8 & 110.4 & 155.0 & 155.0 & 155.0 \\
\hline Manufacturing & 721.8 & 216.9 & 938.8 & 170.2 & 167.1 & 165.2 & 165.7 & 169.0 & 174.5 & 177.6 \\
\hline Food, beverages, and tobacco & 79.8 & 51.0 & 130.9 & 157.1 & 161.1 & 165.9 & 165.7 & 166.5 & 167.5 & 167.5 \\
\hline Textiles & 125.9 & 0.0 & 125.9 & 258.7 & 256.7 & 246.7 & 246.1 & 246.7 & 263.9 & 263.9 \\
\hline Wood and wood products & 9.9 & 20.4 & 30.2 & 171.7 & 171.9 & 168.5 & 164.5 & 163.3 & 162.7 & 162.7 \\
\hline Paper and paper products & 14.2 & 2.0 & 16.1 & 148.9 & 143.5 & 144.6 & 151.0 & 151.7 & 151.4 & 151.4 \\
\hline Chemicals and chemical products & 49.7 & 43.2 & 92.9 & 157.2 & 156.2 & 154.8 & 154.5 & 154.0 & 153.0 & 153.0 \\
\hline Nonmetallic mineral products & 43.7 & 51.2 & 94.9 & 146.8 & 143.2 & 146.3 & 146.9 & 153.4 & 162.9 & 162.9 \\
\hline Basic metal products & 61.6 & 2.4 & 64.0 & 123.2 & 120.0 & 118.9 & 117.0 & 114.0 & 101.2 & 101.2 \\
\hline Fabricated metal products & 327.8 & 46.8 & 374.6 & 163.0 & 156.5 & 153.3 & 155.1 & 162.0 & 169.6 & 169.6 \\
\hline Other & 9.4 & 0.0 & 9.4 & 172.8 & 174.6 & 172.7 & 173.2 & 173.4 & 170.8 & 170.8 \\
\hline
\end{tabular}

Source: Ministry of Planning, Central Statistics Office.

1/ Data cover January-June 2003. 
Table 8. Kuwait: Output of Major Industrial Products, 1997-2002

\begin{tabular}{|c|c|c|c|c|c|c|c|}
\hline Product & Unit & 1997 & 1998 & 1999 & 2000 & 2001 & 2002 \\
\hline Brackish water & billion gallons & 24.0 & 26.1 & 26.1 & 28.2 & 30.1 & 31.0 \\
\hline Potable water & billion gallons & 73.3 & 78.4 & 84.2 & 88.5 & 85.0 & 91.3 \\
\hline Electric energy & bn kwh & 26.7 & 30.0 & 31.6 & 32.3 & 34.5 & 36.4 \\
\hline Detergents & thousand tons & $\ldots$ & $\ldots$ & 0.9 & $\ldots$ & $\ldots$ & ... \\
\hline Sand-lime bricks & thousand cubic meters & 505.7 & 505.2 & 302.8 & 272.0 & 256.0 & ... \\
\hline Cement & thousand tons & $1,370.0$ & $2,309.9$ & 947.3 & $1,187.4$ & $\ldots$ & ... \\
\hline Bran and flour $1 /$ & thousand tons & 196.3 & 195.7 & 202.2 & 210.0 & 211.2 & 225.1 \\
\hline Urea $1 /$ & thousand metric tons & 757.5 & 785.5 & 719.4 & 625.3 & 682.4 & 415.1 \\
\hline Chlorine 1/ & thousand tons & 18.3 & 19.4 & 18.3 & 14.8 & 17.7 & 15.8 \\
\hline Caustic soda $1 /$ & thousand tons & 20.1 & 21.8 & 20.6 & 18.5 & 20.0 & 17.8 \\
\hline Salt 1/ & thousand tons & 42.8 & 41.0 & 38.7 & 36.8 & 37.5 & 35.7 \\
\hline Hydrochloric acid $1 /$ & million gallons & 2.7 & 2.6 & 2.1 & 1.3 & 2.1 & 2.1 \\
\hline Sodium hypochloride 1/ & million cubic meters & 9.2 & 10.9 & 11.6 & 11.7 & 12.7 & 10.7 \\
\hline Hydrogen gas $1 /$ & million cubic meters & 49.8 & 54.5 & 52.7 & 52.3 & 71.9 & 51.3 \\
\hline
\end{tabular}

Source: Ministry of Planning, Central Statistical Office.

1/ For 2002, data cover January-October. 
Table 9. Kuwait: Agricultural and Fisheries Production, 1996/97-2001/02

(In thousands of metric tons)

\begin{tabular}{|c|c|c|c|c|c|c|}
\hline & $1996 / 97$ & $1997 / 98$ & 1998/99 & $1999 / 2000$ & $2000 / 01$ & $2001 / 02$ \\
\hline Vegetable and field crop & 379.2 & 340.9 & 370.1 & 403.3 & 407.8 & 498.1 \\
\hline Wool & 290.0 & 283.0 & 343.0 & 417.0 & 390.0 & 356 \\
\hline Meat & 36.2 & 39.9 & 42.9 & 37.8 & 36.8 & 40.8 \\
\hline Milk & 33.9 & 35.0 & 37.8 & 34.8 & 33.2 & 36.7 \\
\hline Eggs (millions) & 203.5 & 235.5 & 293.1 & 343.5 & 314.6 & 277.4 \\
\hline Fish 1/ & 7.8 & 7.8 & 7.3 & 6.9 & 5.9 & 5.9 \\
\hline
\end{tabular}

Sources: Ministry of Planning, Central Statistical Office; and the Public Authority for Agriculture.

1/ The last four observations are for the calendar years of 1999, 2000, 2001, and 2002, respectively. 
Table 10. Kuwait: Population and Employment, 1997-2002

(In thousands)

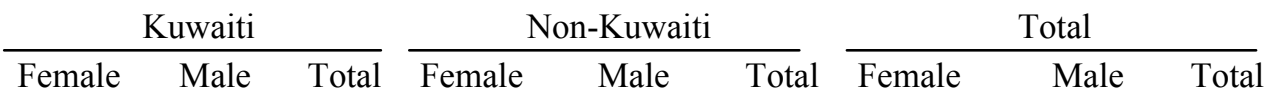

1997

Employed

Total labor force 1/

Population

Participation ratio (in percent)

1998

Employed

Total labor force 1/

Population

Participation ratio (in percent)

1999

Employed

Total labor force 1/

Population

Participation ratio (in percent)

2000

Employed

Total labor force 1/

Population

Participation ratio (in percent)

2001

Employed

Total labor force 1/

Population

Participation ratio (in percent)

2002

Employed

Total labor force 1/

Population

Participation ratio (in percent)

$\begin{array}{rrrrrrrrr}66.3 & 130.5 & 196.8 & 217.7 & 793.4 & 1,011.1 & 284.0 & 923.9 & 1,208.0 \\ 66.8 & 132.4 & 199.2 & 218.7 & 798.7 & 1,017.4 & 285.4 & 931.1 & 1,216.5 \\ 383.1 & 375.8 & 758.9 & 476.6 & 973.3 & 1,449.8 & 859.7 & 1,349.1 & 2,208.8 \\ 17.4 & 35.2 & 26.2 & 45.9 & 82.1 & 70.2 & 33.2 & 69.0 & 55.1\end{array}$

$\begin{array}{rrrrrrrrr}72.6 & 136.8 & 209.4 & 216.2 & 817.5 & 1,033.7 & 288.8 & 954.3 & 1,243.1 \\ 73.0 & 138.5 & 211.6 & 217.4 & 823.1 & 1,040.4 & 290.4 & 961.6 & 1,252.0 \\ 397.3 & 388.7 & 786.0 & 482.1 & 1,002.7 & 1,484.9 & 879.5 & 1,391.4 & 2,270.9 \\ 18.4 & 35.6 & 26.9 & 45.1 & 82.1 & 70.1 & 33.0 & 69.1 & 55.1\end{array}$

$\begin{array}{rrrrrrrrr}75.7 & 143.4 & 219.1 & 210.2 & 787.2 & 997.3 & 285.9 & 930.6 & 1,216.5 \\ 76.2 & 145.1 & 221.4 & 211.5 & 793.2 & 1,004.7 & 287.8 & 938.4 & 1,226.1 \\ 410.8 & 401.4 & 812.3 & 471.8 & 970.9 & 1,442.7 & 882.7 & 1,372.3 & 2,255.0 \\ 18.6 & 36.2 & 27.3 & 44.8 & 81.7 & 69.6 & 32.6 & 68.4 & 54.4\end{array}$

$\begin{array}{rrrrrrrrr}81.7 & 149.1 & 230.8 & 202.3 & 753.9 & 956.2 & 284.1 & 902.9 & 1,187.0 \\ 82.3 & 150.9 & 233.3 & 203.6 & 759.4 & 963.0 & 285.9 & 910.4 & 1,196.3 \\ 426.2 & 415.6 & 841.8 & 448.4 & 927.0 & 1,375.5 & 874.6 & 1,342.6 & 2,217.3 \\ 19.3 & 36.3 & 27.7 & 45.4 & 81.9 & 70.0 & 32.7 & 67.8 & 54.0\end{array}$

$\begin{array}{rrrrrrrrr}88.3 & 155.3 & 243.6 & 228.8 & 785.0 & 1,013.8 & 317.1 & 940.2 & 1,257.4 \\ 91.3 & 158.5 & 249.8 & 230.5 & 790.9 & 1,021.5 & 321.9 & 949.4 & 1,271.3 \\ 441.1 & 429.2 & 870.3 & 478.4 & 960.4 & 1,438.8 & 919.5 & 1,389.6 & 2,309.1 \\ 20.7 & 36.9 & 28.7 & 48.2 & 82.4 & 71.0 & 35.0 & 68.3 & 55.1\end{array}$

$\begin{array}{rrrrrrrrr}95.0 & 160.8 & 255.8 & 247.3 & 843.3 & 1,090.6 & 342.3 & 1,004.2 & 1,346.5 \\ 100.4 & 165.0 & 265.5 & 249.5 & 849.3 & 1,098.8 & 350.0 & 1,014.3 & 1,364.3 \\ 456.0 & 442.3 & 898.3 & 500.7 & 1,020.9 & 1,521.6 & 956.7 & 1,463.2 & 2,419.9 \\ 22.0 & 37.3 & 29.6 & 49.8 & 83.2 & 72.2 & 36.6 & 69.3 & 56.4\end{array}$

Sources: Ministry of Planning, Central Statistical Office; and Civil Information Authority.

1/ Labor force includes population 15 years old and over. 
Table 11. Kuwait: Distribution of Employees by Economic Activity and Nationality, 1999-2002

\begin{tabular}{|c|c|c|c|c|c|c|c|c|c|c|c|c|}
\hline \multirow[b]{2}{*}{ Economic Sectors } & \multicolumn{3}{|c|}{1999} & \multicolumn{3}{|c|}{2000} & \multicolumn{3}{|c|}{2001} & \multicolumn{3}{|c|}{2002} \\
\hline & Kuwaiti & $\begin{array}{c}\text { Non- } \\
\text { Kuwaiti }\end{array}$ & Total & Kuwaiti & $\begin{array}{c}\text { Non- } \\
\text { Kuwaiti }\end{array}$ & Total & Kuwaiti & $\begin{array}{c}\text { Non- } \\
\text { Kuwaiti }\end{array}$ & Total & Kuwaiti & $\begin{array}{c}\text { Non- } \\
\text { Kuwaiti }\end{array}$ & Total \\
\hline
\end{tabular}

\begin{tabular}{|c|c|c|c|c|c|c|c|c|c|c|c|c|}
\hline Agriculture and fisheries & 0.0 & 22.6 & 22.6 & 0.0 & 19.3 & 19.4 & 0.0 & 20.1 & 20.1 & 0.0 & 22.0 & 22.0 \\
\hline Mining and quarrying & 4.1 & 3.4 & 7.6 & 4.3 & 3.0 & 7.3 & 4.4 & 3.0 & 7.4 & 4.6 & 2.9 & 7.5 \\
\hline Manufacturing & 6.8 & 71.1 & 77.9 & 6.9 & 69.4 & 76.4 & 7.1 & 71.7 & 78.8 & 7.2 & 76.4 & 83.6 \\
\hline Construction & 0.7 & 117.6 & 118.3 & 0.8 & 108.3 & 109.1 & 0.9 & 109.1 & 110.1 & 1.0 & 107.3 & 108.3 \\
\hline Electricity, water, and gas & 5.0 & 2.9 & 7.9 & 5.3 & 2.5 & 7.7 & 5.8 & 2.5 & 8.3 & 6.1 & 2.5 & 8.7 \\
\hline Wholesale and retail trade & 2.9 & 193.1 & 196.1 & 3.0 & 198.3 & 201.3 & 3.1 & 209.5 & 212.5 & 3.3 & 216.7 & 220.0 \\
\hline Transportation and communication & 6.0 & 35.6 & 41.6 & 6.1 & 34.4 & 40.5 & 6.0 & 35.0 & 41.0 & 6.2 & 36.8 & 43.0 \\
\hline Finance and business services & 6.3 & 39.6 & 45.8 & 6.6 & 40.7 & 47.3 & 7.5 & 47.2 & 54.7 & 8.1 & 51.0 & 59.1 \\
\hline Public administration & 184.5 & 430.4 & 614.9 & 194.7 & 417.6 & 612.3 & 205.8 & 459.0 & 664.8 & 216.4 & 504.1 & 720.5 \\
\hline Unclassified & 5.1 & 88.4 & 93.5 & 5.6 & 69.4 & 75.0 & 9.2 & 64.5 & 73.6 & 12.6 & 79.1 & 91.7 \\
\hline Total & 221.4 & $1,004.7$ & $1,226.1$ & 233.3 & 963.0 & $1,196.3$ & 249.8 & $1,021.5$ & $1,271.3$ & 265.5 & 1098.8 & 1364.3 \\
\hline & \multicolumn{12}{|c|}{ (In percent of total, by sector) } \\
\hline Agriculture and fisheries & 0.0 & 2.2 & 1.8 & 0.0 & 2.0 & 1.6 & 0.0 & 2.0 & 1.6 & 0.0 & 2.0 & 1.6 \\
\hline Mining and quarrying & 1.9 & 0.3 & 0.6 & 1.8 & 0.3 & 0.6 & 1.8 & 0.3 & 0.6 & 1.7 & 0.3 & 0.6 \\
\hline Manufacturing & 3.1 & 7.1 & 6.4 & 3.0 & 7.2 & 6.4 & 2.8 & 7.0 & 6.2 & 2.7 & 7.0 & 6.1 \\
\hline Construction & 0.3 & 11.7 & 9.6 & 0.4 & 11.2 & 9.1 & 0.4 & 10.7 & 8.7 & 0.4 & 9.8 & 7.9 \\
\hline Electricity, water, and gas & 2.2 & 0.3 & 0.6 & 2.3 & 0.3 & 0.6 & 2.3 & 0.2 & 0.6 & 2.3 & 0.2 & 0.6 \\
\hline Wholesale and retail trade & 1.3 & 19.2 & 16.0 & 1.3 & 20.6 & 16.8 & 1.2 & 20.5 & 16.7 & 1.2 & 19.7 & 16.1 \\
\hline Transportation and communication & 2.7 & 3.5 & 3.4 & 2.6 & 3.6 & 3.4 & 2.4 & 3.4 & 3.2 & 2.3 & 3.3 & 3.2 \\
\hline Finance and business services & 2.8 & 3.9 & 3.7 & 2.8 & 4.2 & 4.0 & 3.0 & 4.6 & 4.3 & 3.1 & 4.6 & 4.3 \\
\hline Public administration & 83.3 & 42.8 & 50.1 & 83.5 & 43.4 & 51.2 & 82.4 & 44.9 & 52.3 & 81.5 & 45.9 & 52.8 \\
\hline Unclassified & 2.3 & 8.8 & 7.6 & 2.4 & 7.2 & 6.3 & 3.7 & 6.3 & 5.8 & 4.7 & 7.2 & 6.7 \\
\hline Total & 100.0 & 100.0 & 100.0 & 100.0 & 100.0 & 100.0 & 100.0 & 100.0 & 100.0 & 100.0 & 100.0 & 100.0 \\
\hline
\end{tabular}


Table 11. Kuwait: Distribution of Employees by Economic Activity and Nationality, 1999-2002

\begin{tabular}{|c|c|c|c|c|c|c|c|c|c|c|c|c|}
\hline \multirow[b]{2}{*}{ Economic Sectors } & \multicolumn{3}{|c|}{1999} & \multicolumn{3}{|c|}{2000} & \multicolumn{3}{|c|}{2001} & \multicolumn{3}{|c|}{2002} \\
\hline & Kuwaiti & $\begin{array}{l}\text { Non- } \\
\text { Kuwaiti }\end{array}$ & Total & Kuwaiti & \multicolumn{2}{|l|}{ Non- } & Kuwaiti & Non- & Total & Kuwaiti & $\begin{array}{l}\text { Non- } \\
\text { Kuwaiti }\end{array}$ & Total \\
\hline & & \multicolumn{11}{|c|}{ (In percent, by nationality) } \\
\hline Agriculture and fisheries & 0.2 & 99.8 & 100.0 & 0.2 & 99.8 & 100.0 & 0.2 & 99.8 & 100.0 & 0.2 & 99.8 & 100.0 \\
\hline Mining and quarrying & 54.5 & 45.5 & 100.0 & 58.8 & 41.2 & 100.0 & 59.9 & 40.1 & 100.0 & 61.3 & 38.7 & 100.0 \\
\hline Manufacturing & 8.7 & 91.3 & 100.0 & 9.1 & 90.9 & 100.0 & 9.0 & 91.0 & 100.0 & 8.6 & 91.4 & 100.0 \\
\hline Construction & 0.6 & 99.4 & 100.0 & 0.8 & 99.2 & 100.0 & 0.9 & 99.1 & 100.0 & 0.9 & 99.1 & 100.0 \\
\hline Electricity, water, and gas & 62.9 & 37.1 & 100.0 & 68.2 & 31.8 & 100.0 & 70.2 & 29.8 & 100.0 & 70.6 & 29.4 & 100.0 \\
\hline Wholesale and retail trade & 1.5 & 98.5 & 100.0 & 1.5 & 98.5 & 100.0 & 1.4 & 98.6 & 100.0 & 1.5 & 98.5 & 100.0 \\
\hline Transportation and communication & 14.4 & 85.6 & 100.0 & 15.0 & 85.0 & 100.0 & 14.7 & 85.3 & 100.0 & 14.4 & 85.6 & 100.0 \\
\hline Finance and business services & 13.7 & 86.3 & 100.0 & 14.0 & 86.0 & 100.0 & 13.7 & 86.3 & 100.0 & 13.7 & 86.3 & 100.0 \\
\hline Public administration & 30.0 & 70.0 & 100.0 & 31.8 & 68.2 & 100.0 & 31.0 & 69.0 & 100.0 & 30.0 & 70.0 & 100.0 \\
\hline Unclassified & 5.4 & 94.6 & 100.0 & 7.4 & 92.6 & 100.0 & 12.4 & 87.6 & 100.0 & 13.7 & 86.3 & 100.0 \\
\hline Total & 18.1 & 81.9 & 100.0 & 19.5 & 80.5 & 100.0 & 19.6 & 80.4 & 100.0 & 19.5 & 80.5 & 100.0 \\
\hline
\end{tabular}

Sources: Ministry of Planning, Central Statistical Office; and Civil Information Authority. 
Table 12. Kuwait: Permits for Expatriate Workers, 1987-2002

(In thousands)

\begin{tabular}{|c|c|c|c|c|}
\hline Year & $\begin{array}{l}\text { Entry Visa } \\
\text { for Work 1/ }\end{array}$ & $\begin{array}{l}\text { Work Permits } \\
\text { Issued First Time }\end{array}$ & $\begin{array}{l}\text { Cancellation of } \\
\text { Work Permits }\end{array}$ & $\begin{array}{c}\text { Net Issuance of } \\
\text { Work Permits }\end{array}$ \\
\hline 1987 & 66.4 & 52.2 & 34.5 & 17.7 \\
\hline 1988 & 89.2 & 61.6 & 23.1 & 48.5 \\
\hline 1989 & 43.1 & 69.9 & 32.8 & 37.1 \\
\hline 1990 & $\ldots$ & $\ldots$ & $\ldots$ & $\ldots$ \\
\hline 1991 & $\ldots$ & $\ldots$ & $\ldots$ & $\ldots$ \\
\hline 1992 & $\ldots$ & $\ldots$ & $\ldots$ & $\ldots$ \\
\hline $19932 /$ & 181.4 & 183.0 & 11.7 & 171.3 \\
\hline $19942 /$ & 52.0 & 78.4 & 32.2 & 46.2 \\
\hline $19952 /$ & 63.4 & 49.3 & 29.1 & 20.2 \\
\hline $19962 /$ & 73.7 & 61.9 & 24.8 & 37.1 \\
\hline $19972 /$ & 21.0 & 17.3 & 4.7 & 12.6 \\
\hline $19982 /$ & 91.1 & 71.4 & 26.6 & 44.8 \\
\hline $19992 /$ & 77.5 & 60.8 & 22.2 & 38.6 \\
\hline $20002 /$ & 58.3 & 40.3 & 5.9 & 34.4 \\
\hline $20012 /$ & 75.6 & 54.9 & $\ldots$ & 54.9 \\
\hline 2002 / & 103.9 & 148.3 & $\ldots$ & 148.3 \\
\hline
\end{tabular}

Sources: Ministry of Social and Labor Affairs (for 1987-93); and Annual Statistical Abstract (from 1994).

1/ Entry visa must be obtained separately from work permit.

2/ These data were compiled on a different basis from those before 1993. 
Table 13. Kuwait: Number of Construction Permits Issued, 1988-2001

\begin{tabular}{|c|c|c|c|}
\hline Year & Residential & Commercial/Industrial & Total \\
\hline 1988 & 2,398 & 40 & 2,438 \\
\hline 1989 & 10,544 & 2,269 & 12,813 \\
\hline 1990 & 4,192 & 792 & 4,984 \\
\hline 1991 & 1,010 & 132 & 1,142 \\
\hline 1992 & 6,145 & 646 & 6,791 \\
\hline 1993 & 13,338 & 1,055 & 14,393 \\
\hline 1994 & 12,955 & 1,459 & 14,414 \\
\hline 1995 & 13,172 & 1,173 & 14,345 \\
\hline 1996 & 14,297 & 1,367 & 15,664 \\
\hline 1997 & 13,700 & 1,447 & 15,147 \\
\hline 1998 & 13,249 & 1,254 & 14,503 \\
\hline 1999 & 12,145 & 1,290 & 13,435 \\
\hline 2000 & 11,608 & 1,716 & 13,324 \\
\hline 2001 & 11,183 & 1,148 & 12,331 \\
\hline
\end{tabular}

Source: Ministry of Planning, Central Statistical Office. 
Table 14. Kuwait: Summary of Government Finance, 1998/99-2003/04

1998/99 1999/2000 2000/01 1/ 2001/02 $\frac{\text { Prel. }}{2002 / 03} \frac{\text { Budget }}{2003 / 04}$

\begin{tabular}{|c|c|c|c|c|c|c|}
\hline \multirow[b]{2}{*}{ Total revenue } & \multicolumn{6}{|c|}{ (In millions of Kuwaiti dinars) } \\
\hline & 3,963 & 7,053 & 8,505 & 6,995 & 7,707 & 3,397 \\
\hline Oil and gas & 2,254 & 4,795 & 6,037 & 4,525 & 5,499 & 2,971 \\
\hline \multicolumn{7}{|l|}{ Investment income and transfer } \\
\hline of profits of public entities $2 /$ & 1,419 & 1,976 & 1,991 & 1,751 & 1,619 & $\ldots$ \\
\hline Other 3/ & 289 & 282 & 476 & 718 & 563 & 392 \\
\hline Total expenditure & 4,161 & 4,050 & 4,279 & 4,729 & 4,848 & 5,666 \\
\hline Current & 3,682 & 3,694 & 3,935 & 4,299 & 4,285 & 4,761 \\
\hline Wages and salaries $4 /$ & 1,490 & 1,498 & 1,573 & 1,636 & 1,706 & 1,834 \\
\hline Goods and services & 882 & 938 & 985 & 1,062 & 1,200 & 1,421 \\
\hline Interest on domestic debt 5/ & 270 & 216 & 249 & 213 & 124 & 49 \\
\hline Interest on foreign debt $2 /$ & 7 & 0 & 0 & 0 & 0 & 0 \\
\hline Transfers abroad & 69 & 84 & 91 & 97 & 61 & 85 \\
\hline Subsidies and transfers & 964 & 958 & 1,036 & 1,291 & 1,194 & 1,372 \\
\hline Capital & 479 & 356 & 344 & 430 & 563 & 905 \\
\hline Of which: land purchases & 0 & 1 & 0 & 4 & 0 & 48 \\
\hline Overall balance & -198 & 3,003 & 4,226 & 2,266 & 2,765 & $-2,270$ \\
\hline Financing & 196 & $-3,003$ & $-4,226$ & $-2,266$ & -2765 & 2270 \\
\hline Domestic (net) & 126 & -457 & -303 & -135 & -162 & $\ldots$ \\
\hline Banks & 78 & -534 & -438 & -169 & -196 & $\ldots$ \\
\hline Nonbanks & 48 & 77 & 135 & 34 & 34 & $\ldots$ \\
\hline External & 0 & 0 & 0 & 0 & 0 & $\ldots$ \\
\hline \multirow[t]{2}{*}{ Reserve funds } & 72 & $-2,546$ & $-3,923$ & $-2,131$ & $-2,603$ & $\ldots$ \\
\hline & \multicolumn{6}{|c|}{ (In percent of GDP) } \\
\hline Revenue & 46.4 & 68.7 & 77.8 & 66.3 & 68.6 & 26.9 \\
\hline Oil and gas & 26.4 & 46.7 & 55.3 & 42.9 & 49.0 & 23.5 \\
\hline Investment income & 16.6 & 19.3 & 18.2 & 16.6 & 14.4 & $\ldots$ \\
\hline Other & 3.4 & 2.7 & 4.4 & 6.8 & 5.0 & 3.1 \\
\hline Expenditure & 48.7 & 39.5 & 39.2 & 44.8 & 43.2 & 44.8 \\
\hline Current & 43.1 & 36.0 & 36.0 & 40.7 & 38.2 & 37.7 \\
\hline Wages and salaries & 17.5 & 14.6 & 14.4 & 15.5 & 15.2 & 14.5 \\
\hline Goods and services & 10.3 & 9.1 & 9.0 & 10.1 & 10.7 & 11.2 \\
\hline Interest on domestic and foreign debt & 3.2 & 2.1 & 2.3 & 2.0 & 1.1 & 0.0 \\
\hline Subsidies and transfers & 11.3 & 9.3 & 9.5 & 12.2 & 10.6 & 10.9 \\
\hline Capital & 5.6 & 3.5 & 3.1 & 4.1 & 5.0 & 7.2 \\
\hline Overall balance & -2.3 & 29.3 & 38.7 & 21.5 & 24.6 & -18.0 \\
\hline \multicolumn{7}{|l|}{ Memorandum items: } \\
\hline \multicolumn{7}{|l|}{ Overall balance (excluding investment } \\
\hline income and profit transfers) & $-1,617$ & 1,027 & 2,235 & 515 & 1,146 & $-2,270$ \\
\hline (In percent of GDP) & -18.9 & 10.0 & 20.5 & 4.9 & 10.2 & -18.0 \\
\hline Kuwait crude export price (US\$ per barrel) & 13.2 & 20.5 & 23.1 & 21.6 & 23.6 & $\ldots$ \\
\hline
\end{tabular}

Sources: Ministry of Finance; Central Bank of Kuwait; and Fund staff estimates.

1/ The fiscal year was shortened to 9 months in 2000/01 FY in preparation for changing the fiscal year from June-July to April-March.

Accordingly, the figures reported for this year were grossed up to 12 months.

2/ Excluded from national budget presentation.

3/ Excludes revenues from utility tariffs (included in the national budget presentation) which are factored in (subtracted) in subsidies and transfers.

4/ For 1999/2000 budget, includes an additional allocation to wages and salaries made on August 15, 1999.

5/ Covers interest on treasury bills and bonds, and on DCP bonds. Only the latter is included in the national budget presentation. 
Table 15. Kuwait: Government Revenue, 1998/99-2003/04

$$
1998 / 99 \quad 1999 / 00 \quad 2000 / 011 / \quad 2001 / 02 \quad \frac{\text { Prel. }}{2002 / 03} \quad \frac{\text { Budget }}{2003 / 04}
$$

\begin{tabular}{|c|c|c|c|c|c|c|}
\hline \multirow[b]{2}{*}{ Total revenue } & \multicolumn{6}{|c|}{ (In millions of Kuwaiti dinars) } \\
\hline & 3,963 & 7,053 & 8,505 & 6,995 & 7,707 & 3,397 \\
\hline Current revenue & 3,962 & 7,053 & 8,505 & 6,994 & 7,681 & 3,363 \\
\hline Oil receipts & 2,254 & 4,795 & 6,037 & 4,525 & 5,499 & 2,971 \\
\hline Investment income and transfer $2 /$ & 1,419 & 1,976 & 1,991 & 1,751 & 1,619 & $\ldots$ \\
\hline Other current revenue & 289 & 282 & 476 & 718 & 563 & 392 \\
\hline Tax revenue & 116 & 97 & 95 & 110 & 135 & 116 \\
\hline \multicolumn{7}{|l|}{ Taxes on income and profits } \\
\hline of non-oil companies & 25 & 17 & 15 & 18 & 26 & 27 \\
\hline Taxes on property transfers & 5 & 4 & 3 & 6 & 8 & 5 \\
\hline Customs duties & 86 & 76 & 77 & 86 & 101 & 84 \\
\hline \multicolumn{7}{|l|}{ Excise taxes } \\
\hline Nontax revenue $3 /$ & 175 & 185 & 381 & 608 & 428 & 276 \\
\hline Capital revenue & 1 & 0 & 0 & 1 & 26 & 34.0 \\
\hline & \multicolumn{6}{|c|}{ (In percent of total revenue) } \\
\hline Oil receipts & 56.9 & 68.0 & 71.0 & 64.7 & 71.4 & 88.3 \\
\hline Investment income & 35.8 & 28.0 & 23.4 & 25.0 & 21.4 & 0.0 \\
\hline Tax revenue & 2.9 & 1.4 & 1.1 & 1.6 & 1.7 & 3.4 \\
\hline \multicolumn{7}{|l|}{ Of which } \\
\hline Customs duties & 2.2 & 1.1 & 0.9 & 1.2 & 1.3 & 2.5 \\
\hline Nontax revenue & 4.4 & 2.6 & 4.5 & 8.7 & 5.5 & 8.1 \\
\hline
\end{tabular}

Sources: Ministry of Finance; and Fund staff estimates.

1/ The fiscal year was shortened to 9 months in 2000/01 FY in preparation for changing the fiscal year from June-July to April-March. Accordingly, the figures reported for this year were grossed up to 12 months.

$2 /$ Income from government's external assets; excluded from national budget and ex-post fiscal accounts.

3/ Includes net surplus of PTT. 
Table 16. Kuwait: Government Current Expenditure, 1998/99-2003/04

\begin{tabular}{|c|c|c|c|c|c|c|}
\hline & 1998/99 & $1999 / 00$ & $2000 / 011 /$ & $2001 / 02$ & $\frac{\text { Prel. }}{2002 / 03}$ & $\frac{\text { Budget }}{2003 / 04}$ \\
\hline & \multicolumn{6}{|c|}{ (In millions of Kuwaiti dinars) } \\
\hline \multicolumn{7}{|l|}{ Economic classification } \\
\hline Total current expenditure & 3,682 & 3,694 & 3,935 & 4,299 & 4,285 & 4,761 \\
\hline Wages and salaries 2/ & 1,490 & 1,498 & 1,573 & 1,636 & 1,706 & 1,834 \\
\hline Goods and non-interest services & 882 & 938 & 985 & 1,062 & 1,200 & 1,421 \\
\hline Interest on domestic debt & 270 & 216 & 249 & 213 & 124 & 49 \\
\hline Interest on DCP bonds & 123 & 100 & 84 & 61 & 31 & 49 \\
\hline Interest on treasury bills 3 / & 57 & 0 & 44 & 36 & 32 & $\ldots$ \\
\hline Interest on treasury bonds 3 / & 89 & 116 & 121 & 116 & 61 & ... \\
\hline Interest on foreign debt $3 /$ & 7 & 0 & 0 & 0 & 0 & 0 \\
\hline Transfers abroad & 69 & 84 & 91 & 97 & 61 & 85 \\
\hline Subsidies and domestic transfers & 964 & 958 & 1,045 & 1,291 & 1,194 & 1,372 \\
\hline \multicolumn{7}{|l|}{ Functional classification } \\
\hline Total current expenditure 4/ & 3,682 & 3,694 & 3,935 & 4,299 & 4,285 & 4,761 \\
\hline General public service & 297 & 324 & 373 & 364 & 362 & 405 \\
\hline Defense & 683 & 710 & 708 & 781 & 888 & 1,049 \\
\hline Public order & 376 & 385 & 401 & 422 & 449 & 471 \\
\hline Education & 578 & 579 & 605 & 632 & 643 & 698 \\
\hline Health & 275 & 266 & 273 & 298 & 310 & 341 \\
\hline Social affairs & 691 & 758 & 801 & 993 & 838 & 837 \\
\hline Social security & 475 & 456 & 476 & 471 & 535 & 551 \\
\hline Social welfare & 216 & 303 & 325 & 522 & 303 & 286 \\
\hline Housing and utilities & 119 & 198 & 128 & 103 & 105 & 151 \\
\hline Economic services & 141 & 210 & 283 & 367 & 300 & 481 \\
\hline Mining, manufacturing, and construction & 14 & 12 & 13 & 12 & 13 & 14 \\
\hline Electricity, etc. & 73 & 142 & 220 & 307 & 234 & 381 \\
\hline Agriculture & 17 & 17 & 17 & 14 & 23 & 24 \\
\hline Other & 37 & 39 & 32 & 34 & 30 & 62 \\
\hline Other & 522 & 264 & 361 & 187 & 203 & 328 \\
\hline Economic classification & \multicolumn{6}{|c|}{ (In percent of total) } \\
\hline Of which & & & & & & \\
\hline Wages and salaries & 40.5 & 40.6 & 40.0 & 38.1 & 39.8 & 38.5 \\
\hline Goods and services & 24.0 & 25.4 & 25.0 & 24.7 & 28.0 & 29.8 \\
\hline Transfers abroad & 1.9 & 2.3 & 2.3 & 2.3 & 1.4 & 1.8 \\
\hline Subsidies and domestic transfers & 26.2 & 25.9 & 26.6 & 30.0 & 27.9 & 28.8 \\
\hline \multirow{2}{*}{\multicolumn{7}{|c|}{$\begin{array}{l}\text { Functional classification } \\
\text { Of which }\end{array}$}} \\
\hline & & & & & & \\
\hline General public service & 8.1 & 8.8 & 9.5 & 8.5 & 8.4 & 8.5 \\
\hline Defense & 18.6 & 19.2 & 18.0 & 18.2 & 20.7 & 22.0 \\
\hline Health & 7.5 & 7.2 & 6.9 & 6.9 & 7.2 & 7.2 \\
\hline Housing and utilities & 3.2 & 5.4 & 3.3 & 2.4 & 2.5 & 3.2 \\
\hline Economic services & 3.8 & 5.7 & 7.2 & 8.5 & 7.0 & 10.1 \\
\hline
\end{tabular}

Sources: Ministry of Finance; and Fund staff estimates.

1/ The fiscal year was shortened to 9 months in 2000/01 FY in preparation for changing the fiscal year from June-July 1 April-March. Accordingly, the figures reported for this year were grossed up to 12 months.

2/ Excludes military wages and salaries.

3/ Excluded from national budget presentation.

4/ Totals may differ from the sum of the components because the authorities do not include interest payments on treasury bills and bonds in their presentation. 
Table 17. Kuwait: Government Capital Expenditures and Land Purchases, 1998/99-2003/04

\begin{tabular}{|c|c|c|c|c|c|c|}
\hline & 1998/99 & 1999/00 & $2000 / 011 /$ & $2001 / 02$ & $\frac{\text { Prel. }}{2002 / 03}$ & $\frac{\text { Budget }}{2003 / 04}$ \\
\hline & \multicolumn{6}{|c|}{ (In millions of Kuwaiti dinars) } \\
\hline Capital expenditure & 479 & 356 & 344 & 430 & 563 & 905 \\
\hline General public services & 74 & 27 & 32 & 30 & 63 & 73 \\
\hline Defense & 3 & 2 & -1 & 3 & 3 & 8 \\
\hline Education & 40 & 34 & 47 & 48 & 58 & 102 \\
\hline Health & 14 & 13 & 20 & 27 & 20 & 39 \\
\hline Social affairs & 4 & 5 & 4 & 7 & 11 & 12 \\
\hline Housing & 62 & 67 & 63 & 77 & 136 & 176 \\
\hline Economic services & 266 & 161 & 133 & 186 & 226 & 348 \\
\hline \multicolumn{7}{|l|}{ Of which } \\
\hline Electricity and water & 237 & 147 & 116 & 169 & 209 & 313 \\
\hline Roads & 13 & $\ldots$ & 0 & $\ldots$ & 0 & \\
\hline Other & 16 & 14 & 17 & 17 & 17 & 35 \\
\hline Public order & 16 & 19 & 32 & 30 & 26 & 38 \\
\hline Other $2 /$ & 13 & 26 & 15 & 18 & 20 & 61 \\
\hline Land purchases & 0 & 1 & 0 & 4 & 0 & 48 \\
\hline Capital and land, total & 479 & 356 & 344 & 430 & 563 & 905 \\
\hline & \multicolumn{6}{|c|}{ (In percent of total) } \\
\hline Capital expenditure & 100.0 & 100.0 & 100.0 & 100.0 & 100.0 & 100.0 \\
\hline \multicolumn{7}{|l|}{ Of which } \\
\hline General public services & 15.4 & 7.6 & 9.3 & 7.0 & 11.2 & 8.1 \\
\hline Education & 8.4 & 9.5 & 13.6 & 11.2 & 10.3 & 11.3 \\
\hline Health & 2.9 & 3.6 & 5.8 & 6.3 & 3.6 & 4.3 \\
\hline Housing & 12.9 & 18.8 & 18.2 & 17.9 & 24.2 & 19.4 \\
\hline Economic services & 55.5 & 45.2 & 38.8 & 43.3 & 40.1 & 38.5 \\
\hline Land purchases & 0.0 & 0.4 & 0.0 & 0.9 & 0.0 & 5.3 \\
\hline Total & 100.0 & 100.0 & 100.0 & 100.0 & 100.0 & 100.0 \\
\hline
\end{tabular}

Sources: Ministry of Finance; and Fund staff estimates.

1/ The fiscal year was shortened to 9 months in 2000/01 FY in preparation for changing the fiscal year from June-July to April-March. Accordingly, the figures reported for this year were grossed up to 12 months.

2 / Includes contingency funds not allocated to specific expenditures. 
Table 18. Kuwait: Government Domestic Subsidies and Transfers, 1998/99-2003/04

(In millions of Kuwaiti dinars)

$$
1998 / 99 \quad 1999 / 00 \quad 2000 / 011 / \quad 2001 / 02 \quad \frac{\text { Prel. }}{2002 / 03} \frac{\text { Budget }}{2003 / 04}
$$

Total current subsidies

Food and commodity subsidies

Petroleum product subsidies

Electricity and water

\section{1}

9

5

107

Total transfers

Social Security Institute

Individuals

Of which

End of employment benefits

Cancellation of housing loans

Private domestic institutions

Other public entities

Other

Total current subsidies and transfers

843

467

176

32

35

Memorandum items:

Subsidies and transfers
As percent of GDP
As percent of government expenditure

274
6
6
262

684

442

160

16

$$
33
$$

$\begin{array}{rr}8 & 10 \\ 144 & 40\end{array}$

40

32

958

1,045

1,291

1,194

1372

11.3
23.2
9.6

24.4
12.2

27.3
446

8

7

431

926

551

223

15

35

11

54

87

30

Sources: Ministry of Finance; and Fund staff estimates.

1/ The fiscal year was shortened to 9 months in 2000/01 FY in preparation for changing the fiscal year from June-July to April-March. Accordingly, the figures reported for this year were grossed up to 12 months. 
Table 19. Kuwait: Monetary Survey, 1997-2003

\begin{tabular}{|c|c|c|c|c|c|c|c|}
\hline End of Period & 1997 & 1998 & 1999 & 2000 & 2001 & 2002 & $\frac{\text { Sept. 1/ }}{2003}$ \\
\hline & \multicolumn{7}{|c|}{ (In millions of Kuwaiti dinars) } \\
\hline Foreign assets (net) & 1,929 & 1,784 & 1,899 & 2,778 & 3,307 & 2,930 & 2,647 \\
\hline Central bank & 1,034 & 1,080 & 1,320 & 2,005 & 2,850 & 2,521 & 2,002 \\
\hline Local banks & 895 & 704 & 579 & 773 & 457 & 409 & 645 \\
\hline Domestic assets (net) & 5,687 & 5,773 & 5,780 & 5,386 & 5,902 & 6,716 & 8,044 \\
\hline Claims on government (net) & 3,782 & 3,678 & 3,475 & 2,911 & 2,573 & 2,595 & 2,357 \\
\hline Central bank (net) & -237 & -205 & -392 & -532 & -598 & -354 & -404 \\
\hline Claims & 39 & 0 & 45 & 0 & 0 & 0 & 0 \\
\hline Deposits (increase -) & 276 & 205 & 437 & 532 & 598 & 354 & 404 \\
\hline Local banks (net) & 4,019 & 3,883 & 3,867 & 3,443 & 3,171 & 2,949 & 2,761 \\
\hline Claims & 4,363 & 4,140 & 4,062 & 3,628 & 3,402 & 3,248 & 3,077 \\
\hline Government debt bonds & 2,389 & 2,246 & 1,931 & 1,491 & 1,294 & 1,006 & 885 \\
\hline Public debt instruments & 1,973 & 1,894 & 2,132 & 2,137 & 2,108 & 2,242 & 2,192 \\
\hline Other claims & 0 & 0 & 0 & 0 & 0 & 0 & 0 \\
\hline Deposits (increase -) & 344 & 257 & 195 & 185 & 231 & 299 & 316 \\
\hline Claims on nongovernment sector & 4,745 & 5,303 & 5,572 & 5,871 & 6,851 & 7,824 & 9,301 \\
\hline Credit facilities & 4,324 & 4,802 & 5,015 & 5,252 & 6,125 & 6,954 & 8,391 \\
\hline Local investments & 420 & 501 & 557 & 619 & 726 & 870 & 910 \\
\hline Other items (net) & $-2,840$ & $-3,209$ & $-3,268$ & $-3,396$ & $-3,522$ & $-3,703$ & $-3,614$ \\
\hline Broad money & 7,616 & 7,557 & 7,678 & 8,163 & 9,209 & 9,646 & 10,691 \\
\hline Money & 1,247 & 1,143 & 1,371 & 1,468 & 1,641 & 2,067 & 2,598 \\
\hline Quasi money, of which: & 6,368 & 6,413 & 6,307 & 6,695 & 7,567 & 7,580 & 8,093 \\
\hline Foreign currency deposits & 1,154 & 1,037 & 881 & 895 & 892 & 904 & 1,124 \\
\hline
\end{tabular}

(Annual percentage change)

Foreign assets (net)

Central bank

Local banks

Domestic assets (net)

Claims on government (net)

Central bank (net)

Claims

Deposits (increase $=+$ )

Local banks (net)

Claims on nongovernment (net)

Credit facilites

Local investments

Other items (net)
$-21$

$-1.4$

$-36.4 \quad-21.4$

16.7

$-5.0$

$-8.3$

$-4.6$

6.3

$-4.3$

35.5

36.3

27.5

$-8.9$

\section{4}

22.2

$-17.7$

1.5

$-2.7$

13.5

$-99.7$

$-25.8$

$-3.4$

11.8

11.0

19.3

$-13.0$

0.1

$-5.5$

$-91.3$

113.2

$-0.4$

5.1

4.4

11.2

$-1.8$
46.3

51.9

33.5

$-6.8$

$-16.2$

$-35.5$

$-100.0$
21.7

$-11.0$

5.4

4.7

11.0

$-3.9$
19.0

42.2

$-40.9$

9.6

$-11.6$

$-12.4$

...

12.3

$-7.9$

16.7

16.6

17.3

$-3.7$
$-11.4$

$-9.7$

$-11.6$

$-20.6$

57.6

13.8

19.8

0.8

$-9.2$

40.7

14.1

...

$-40.7$

14.1

$-7.0$

$-6.4$

14.2

18.9

13.5

20.7

19.9

4.6

$-5.1$ 
Table 19. Kuwait: Monetary Survey, 1997-2003

\begin{tabular}{|c|c|c|c|c|c|c|c|}
\hline \multirow{3}{*}{ End of Period } & & & & & & & Sept. 1/ \\
\hline & 1997 & 1998 & 1999 & 2000 & 2001 & 2002 & 2003 \\
\hline & \multicolumn{7}{|c|}{ (Annual percentage change) } \\
\hline Broad money & 3.9 & -0.8 & 1.6 & 6.3 & 12.8 & 4.8 & 10.8 \\
\hline Money & 0.4 & -8.3 & 19.9 & 7.0 & 11.8 & 25.9 & 25.7 \\
\hline Quasi money, of which: & 4.6 & 0.7 & -1.7 & 6.2 & 13.0 & 0.2 & 6.8 \\
\hline Foreign currency deposits & -8.2 & -10.1 & -15.1 & 1.6 & -0.3 & 1.3 & 24.4 \\
\hline
\end{tabular}

(Change in percent of broad money stock a year earlier)

\begin{tabular}{|c|c|c|c|c|c|c|c|}
\hline Foreign assets (net) & -7.2 & -1.9 & 1.5 & 11.4 & 6.5 & -4.1 & -2.9 \\
\hline Central bank & -0.2 & 0.6 & 3.2 & 8.9 & 10.4 & -3.6 & -5.4 \\
\hline Local banks & -7.0 & -2.5 & -1.7 & 2.5 & -3.9 & -0.5 & 2.4 \\
\hline Domestic assets (net) & 11.1 & 1.1 & 0.1 & -5.1 & 6.3 & 8.8 & 13.8 \\
\hline Claims on government (net) & -2.7 & -1.4 & -2.7 & -7.3 & -4.1 & 0.2 & -2.5 \\
\hline Central bank (net) & -0.2 & 0.4 & -2.5 & -1.8 & -0.8 & 2.6 & -0.5 \\
\hline Local banks (net) & -2.5 & -1.8 & -0.2 & -5.5 & -3.3 & -2.4 & -1.9 \\
\hline Government debt bonds & -6.2 & -1.9 & -4.2 & -5.7 & -2.4 & -3.1 & -1.3 \\
\hline Claims on nongovernment sector & 16.9 & 7.3 & 3.6 & 3.9 & 12.0 & 10.6 & 15.3 \\
\hline Other items (net) & -3.2 & -4.8 & -0.8 & -1.7 & -1.5 & -2.0 & 0.9 \\
\hline Broad money & 3.9 & -0.8 & 1.6 & 6.3 & 12.8 & 4.8 & 10.8 \\
\hline Money & 0.1 & -1.4 & 3.0 & 1.3 & 2.1 & 4.6 & 5.5 \\
\hline Quasi money, of which: & 3.8 & 0.6 & -1.4 & 5.1 & 10.7 & 0.1 & 5.3 \\
\hline Foreign currency deposits & -1.4 & -1.5 & -2.1 & 0.2 & 0.0 & 0.1 & 2.3 \\
\hline
\end{tabular}

Source: Central Bank of Kuwait.

1/ For 2003, percentage change for January-September 2003 
Table 20. Kuwait: Monetary Accounts of the Central Bank, 1997-2003

(In millions of Kuwaiti dinars)

\begin{tabular}{|c|c|c|c|c|c|c|}
\hline & & & & & & Sept. \\
\hline End of Period & 1998 & 1999 & 2000 & 2001 & 2002 & 2003 \\
\hline Foreign assets $1 /$ & 1,082 & 1,322 & 2,009 & 2,854 & 2,533 & 2,022 \\
\hline Gold & 32 & 32 & 32 & 32 & 32 & 32 \\
\hline Other foreign assets & 1,051 & 1,291 & 1,977 & 2,822 & 2,501 & 1,990 \\
\hline Rediscounted commercial paper & $\ldots$ & $\ldots$ & $\ldots$ & $\ldots$ & $\ldots$ & 0 \\
\hline Deposits with local banks & $\ldots$ & $\ldots$ & 14 & $\ldots$ & $\ldots$ & 0 \\
\hline Claims on government & $\ldots$ & 45 & $\ldots$ & $\ldots$ & $\ldots$ & 0 \\
\hline Unclassified assets & 17 & 28 & 47 & 66 & 53 & 26 \\
\hline Total assets $=$ Total liabilities & 1,100 & 1,395 & 2,069 & 2,920 & 2,585 & 2,048 \\
\hline Reserve money & 448 & 583 & 535 & 521 & 590 & 581 \\
\hline Currency in circulation & 349 & 443 & 417 & 401 & 442 & 463 \\
\hline Currency with banks & 64 & 94 & 89 & 56 & 82 & 57 \\
\hline Local banks' deposits with CBK & 36 & 46 & 29 & 64 & 66 & 61 \\
\hline Foreign liabilities $2 /$ & 2 & 3 & 4 & 4 & 12 & 20 \\
\hline Government deposits & 205 & 437 & 532 & 598 & 354 & 404 \\
\hline Capital accounts & 184 & 184 & 184 & 184 & 184 & 197 \\
\hline Unclassified liabilities & 185 & 167 & 161 & 214 & 252 & 258 \\
\hline Local banks deposits & 75 & 22 & 655 & 1,399 & 1,193 & 588 \\
\hline \multicolumn{7}{|l|}{ Memorandum items: } \\
\hline Net foreign assets & 1,080 & 1,320 & 2,005 & 2,850 & 2,521 & 2,002 \\
\hline Currency issued & 412 & 537 & 505 & 457 & 524 & 520 \\
\hline
\end{tabular}

Source: Central Bank of Kuwait.

1/ Excludes SDRs and IMF reserve position.

2/ Accounts of international organizations. 
Table 21. Kuwait: Balance Sheet of the Local Banks, 1997-2003 1/

(In millions of Kuwaiti dinars)

\begin{tabular}{|c|c|c|c|c|c|c|c|}
\hline End of Period & 1997 & 1998 & 1999 & 2000 & 2001 & 2002 & $\frac{\text { Sept. }}{2003}$ \\
\hline Reserves & 79 & 98 & 141 & 119 & 120 & 144 & 115 \\
\hline Cash & 66 & 64 & 94 & 89 & 56 & 82 & 57 \\
\hline Balances with central bank & 13 & 34 & 47 & 31 & 64 & 62 & 58 \\
\hline Foreign assets & 2,118 & 1,788 & 1,787 & 1,968 & 2,027 & 2,441 & 2,417 \\
\hline Claims on nongovernment sector & 4,745 & 5,303 & 5,573 & 5,871 & 6,851 & 7,824 & 9,301 \\
\hline Credit facilities & 4,324 & 4,802 & 5,015 & 5,252 & 6,125 & 6,954 & 8,391 \\
\hline Local investments & 420 & 501 & 557 & 619 & 726 & 870 & 910 \\
\hline Claims on government & 4,363 & 4,140 & 4,062 & 3,628 & 3,402 & 3,248 & 3,077 \\
\hline Government debt bonds 2/ & 2,389 & 2,246 & 1,931 & 1,491 & 1,294 & 1,006 & 885 \\
\hline Public debt instruments $3 /$ & 1,973 & 1,894 & 2,132 & 2,137 & 2,108 & 2,242 & 2,192 \\
\hline Other claims & 0 & 0 & 0 & 0 & 0 & 0 & 0 \\
\hline Interbank deposits & 973 & 1,088 & 988 & 1,113 & 890 & 1,825 & 2,824 \\
\hline Time deposits with CBK & 40 & 75 & 7 & 655 & 1,399 & 1,193 & 589 \\
\hline Other assets & 374 & 383 & 359 & 452 & 377 & 389 & 479 \\
\hline Total assets $=$ Total liabilities & 12,690 & 12,875 & 12,917 & 13,806 & 15,064 & 17,064 & 18,802 \\
\hline Broad money & 7,271 & 7,208 & 7,235 & 7,746 & 8,807 & 9,204 & 10,228 \\
\hline Demand deposits & 902 & 795 & 929 & 1,051 & 1,240 & 1,625 & 2,135 \\
\hline Quasi-money deposits & 6,368 & 6,413 & 6,307 & 6,696 & 7,567 & 7,580 & 8,093 \\
\hline Savings & 1,116 & 1,144 & 1,084 & 1,257 & 1,394 & 1,536 & 1,714 \\
\hline Time & 4,065 & 4,166 & 4,261 & 4,449 & 5,217 & 5,139 & 5,088 \\
\hline Foreign currency & 1,154 & 1,037 & 881 & 895 & 892 & 904 & 1,124 \\
\hline Certificates of deposit & 34 & 66 & 81 & 95 & 64 & 0 & 167 \\
\hline Government deposits & 344 & 257 & 195 & 185 & 231 & 299 & 316 \\
\hline Foreign liabilities & 1,222 & 1,084 & 1,208 & 1,195 & 1,571 & 2,032 & 1,772 \\
\hline Interbank deposits & 1,043 & 1,292 & 1,249 & & 1,254 & 2,218 & 2,904 \\
\hline Own funds & 1,408 & 1,470 & 1,504 & 1,583 & 1,682 & 1,770 & 1,914 \\
\hline Other liabilities & 1,402 & 1,565 & 1,525 & 1,734 & 1,520 & 1,540 & 1,668 \\
\hline
\end{tabular}

Source: Central Bank of Kuwait.

1/ Includes 7 commercial banks, 2 specialized banks, and Kuwait Finance House.

2/ Includes the purchase by the government of the real estate portfolio of the Kuwait Finance House.

3/ Primarily treasury bills and bonds. 
Table 22. Kuwait: Distribution of Local Bank Credit Outstanding to the Private Sector, 1997-2003

(In millions of Kuwaiti dinars)

Trade

Industry

Construction

Agriculture and fisheries

Financial institutions

Personal facilities

Of which: credit for trading in securities

Real estate

Other

Total

Trade

Industry

Construction

Agriculture and fisheries

Financial institutions

Personal facilities

Of which: credit for trading in securities

Real estate

Other

Total

Trade

Industry

Construction

Agriculture and fisheries

Financial institutions

Personal facilities

Of which: credit for trading in securities

Real estate

Other

Total

$\begin{array}{rrrrrrr}706 & 818 & 930 & 968 & 1,076 & 1,022 & 1,031 \\ 373 & 373 & 389 & 397 & 440 & 533 & 553 \\ 304 & 394 & 363 & 442 & 377 & 480 & 568 \\ 16 & 9 & 8 & 11 & 14 & 20 & 41 \\ 265 & 281 & 305 & 330 & 712 & 639 & 1,016 \\ 1,722 & 1,716 & 1,602 & 1,684 & 2,023 & 2,602 & 3,171 \\ 333 & 292 & 217 & 249 & 386 & 398 & 560 \\ 674 & 843 & 1,000 & 854 & 1,165 & 1,298 & 1,389 \\ 264 & 368 & 418 & 567 & 319 & 360 & 622 \\ 4,324 & 4,802 & 5,015 & 5,252 & 6,125 & 6,954 & 8,391\end{array}$

(In percent of total)

$\begin{array}{rrrrrrr}16.3 & 17.0 & 18.5 & 18.4 & 17.6 & 14.7 & 12.3 \\ 8.6 & 7.8 & 7.8 & 7.6 & 7.2 & 7.7 & 6.6 \\ 7.0 & 8.2 & 7.2 & 8.4 & 6.1 & 6.9 & 6.8 \\ 0.4 & 0.2 & 0.2 & 0.2 & 0.2 & 0.3 & 0.5 \\ 6.1 & 5.8 & 6.1 & 6.3 & 11.6 & 9.2 & 12.1 \\ 39.8 & 35.7 & 31.9 & 32.1 & 33.0 & 37.4 & 37.8 \\ 7.7 & 6.1 & 4.3 & 4.7 & 6.3 & 5.7 & 6.7 \\ 15.6 & 17.5 & 19.9 & 16.3 & 19.0 & 18.7 & 16.6 \\ 6.1 & 7.7 & 8.3 & 10.8 & 5.2 & 5.2 & 7.4 \\ 100.0 & 100.0 & 100.0 & 100.0 & 100.0 & 100.0 & 100.0\end{array}$

(Changes in percent)

$\begin{array}{rrrrrrr}36.0 & 16.0 & 13.6 & 4.1 & 11.2 & -5.1 & 0.9 \\ 65.6 & 0.0 & 4.3 & 2.0 & 10.8 & 21.1 & 3.8 \\ 46.9 & 29.6 & -7.9 & 21.5 & -14.7 & 27.4 & 18.3 \\ 60.9 & -44.2 & -5.1 & 26.3 & 31.0 & 44.6 & 105.0 \\ 44.1 & 6.0 & 8.6 & 8.1 & 115.9 & -10.2 & 59.0 \\ 18.2 & -0.4 & -6.6 & 5.1 & 20.1 & 28.7 & 21.9 \\ 31.9 & -12.4 & -25.6 & 14.3 & 55.2 & 3.2 & 40.7 \\ 60.3 & 25.0 & 18.7 & -14.6 & 36.5 & 11.4 & 7.0 \\ 74.9 & 39.3 & 13.6 & 35.8 & -43.7 & 12.8 & 72.8 \\ 36.3 & 11.0 & 4.4 & 4.7 & 16.6 & 13.5 & 20.7\end{array}$

Source: Central Bank of Kuwait.

1/ For 2003, percentage changes are for January-September. 
Table 23. Kuwait: Structure of Interest Rates, 1997-2003 1/

(In percent per annum)

\begin{tabular}{|c|c|c|c|c|c|c|c|c|c|c|c|}
\hline \multirow{2}{*}{\multicolumn{2}{|c|}{ Valid Since }} & \multirow{3}{*}{$\begin{array}{c}\text { Discount } \\
\text { Rate }\end{array}$} & \multicolumn{5}{|c|}{ Maximum Lending Rates } & \multicolumn{4}{|c|}{ Lending Margin Over Discount Rate } \\
\hline & & & \multirow{2}{*}{$\begin{array}{l}\text { Consumer } \\
\text { Loans }\end{array}$} & \multicolumn{2}{|c|}{$\begin{array}{c}\text { Credit One Year } \\
\text { or Less }\end{array}$} & \multicolumn{2}{|c|}{$\begin{array}{r}\text { Credit More } \\
\text { Than One Year }\end{array}$} & \multicolumn{2}{|c|}{$\begin{array}{c}\text { Credit One Year } \\
\text { or Less }\end{array}$} & \multicolumn{2}{|c|}{$\begin{array}{l}\text { Credit More } \\
\text { Than One Year }\end{array}$} \\
\hline Year & Month & & & Overdraft & Loans & Overdraft & Loans & Overdraft & Loans & Overdraft & Loans \\
\hline 1997 & 11 & 7.50 & 7.50 & 10.50 & 10.00 & 11.50 & 11.50 & 3.00 & 2.50 & 4.00 & 4.00 \\
\hline 1998 & 11 & 7.00 & 7.00 & 10.00 & 9.50 & 11.00 & 11.00 & 3.00 & 2.50 & 4.00 & 4.00 \\
\hline 1999 & 3 & 6.75 & 6.75 & 9.75 & 9.25 & 10.75 & 10.75 & 3.00 & 2.50 & 4.00 & 4.00 \\
\hline 2000 & 5 & 7.25 & 7.25 & 10.25 & 9.75 & 11.25 & 11.25 & 3.00 & 2.50 & 4.00 & 4.00 \\
\hline 2001 & 1 & 6.75 & 6.75 & 9.75 & 9.25 & 10.75 & 10.75 & 3.00 & 2.50 & 4.00 & 4.00 \\
\hline 2001 & 2 & 6.25 & 6.25 & 9.25 & 8.75 & 10.25 & 10.25 & 3.00 & 2.50 & 4.00 & 4.00 \\
\hline 2001 & 3 & 5.75 & 5.75 & 8.75 & 8.25 & 9.75 & 9.75 & 3.00 & 2.50 & 4.00 & 4.00 \\
\hline 2001 & 5 & 5.50 & 5.50 & 8.50 & 8.00 & 9.50 & 9.50 & 3.00 & 2.50 & 4.00 & 4.00 \\
\hline 2001 & 8 & 5.25 & 5.25 & 8.25 & 7.75 & 9.25 & 9.25 & 3.00 & 2.50 & 4.00 & 4.00 \\
\hline 2001 & 9 & 4.75 & 4.75 & 7.75 & 7.25 & 8.75 & 8.75 & 3.00 & 2.50 & 4.00 & 4.00 \\
\hline 2001 & 10 & 4.25 & $\begin{array}{l}4.25 \\
\text { v.vu }\end{array}$ & 7.25 & 6.75 & 8.25 & 8.25 & 3.00 & 2.50 & 4.00 & 4.00 \\
\hline 2002 & 6 & 3.75 & 3.75 & 6.75 & 6.25 & 7.75 & 7.75 & 3.00 & 2.50 & 4.00 & 4.00 \\
\hline 2002 & 11 & 3.25 & 3.25 & 6.25 & 5.75 & 7.25 & 7.25 & 3.00 & 2.50 & 4.00 & 4.00 \\
\hline
\end{tabular}

Memorandum items:

\section{Types of Rates}

One week REPO rate

Overnight rate

Sales/purchase of treasury bills rate

\section{Market Rates}

Interbank rate 1/

Savings deposit rate

Time deposit rate $2 /$

Commercial loans rate

One year or less

Ceiling

More than one year

Ceiling

Consumer loans rate

\section{Rate Formation}

Discount rate +0.50

REPO rate. +0.125 percentage point

Variable rate set daily by CBK.
One week; one-, three-, six-, nine-, and twelve-month rate.

Variable rate; floor abolished effective February 1995.

Variable rate; floor abolished effective February 1995.

Market Rate

Discount rate +2.5 percentage points

Market Rate

Discount rate +4 percentage points.

Market Rate

Discount rate, but front loaded. 
Table 24. Kuwait: Interest Rates on Kuwaiti Dinar and U.S. Dollar Deposits with Local Banks, 1997-2003

(In percent per annum, period average)

\begin{tabular}{|c|c|c|c|c|c|c|}
\hline & \multicolumn{2}{|c|}{$\begin{array}{c}\text { Kuwaiti Dinar } \\
\text { Deposits }\end{array}$} & \multicolumn{2}{|c|}{$\begin{array}{l}\text { U.S. Dollar } \\
\text { Deposits }\end{array}$} & \multicolumn{2}{|c|}{$\begin{array}{l}\text { Interest Differential } \\
\text { KD minus U.S. Dollar }\end{array}$} \\
\hline & 3 months & 6 months & 3 months & 6 months & 3 months & 6 months \\
\hline 1997 & 5.91 & 6.09 & 5.25 & 5.34 & 0.66 & 0.75 \\
\hline 1998 & 5.87 & 6.12 & 5.08 & 5.07 & 0.79 & 1.05 \\
\hline 1999 & 5.27 & 5.53 & 4.86 & 4.96 & 0.41 & 0.58 \\
\hline 2000 & 5.43 & 5.70 & 5.99 & 6.12 & -0.57 & -0.42 \\
\hline 2001 & 3.70 & 3.86 & 3.33 & 3.28 & 0.37 & 0.58 \\
\hline 2002 & 2.21 & 2.34 & 1.32 & 1.40 & 0.89 & 0.94 \\
\hline \multicolumn{7}{|l|}{1998} \\
\hline Q1 & 5.95 & 6.14 & 5.18 & 5.21 & 0.77 & 0.93 \\
\hline Q2 & 5.90 & 6.19 & 5.22 & 5.27 & 0.68 & 0.92 \\
\hline Q3 & 5.85 & 6.12 & 5.18 & 5.19 & 0.68 & 0.94 \\
\hline Q4 & 5.77 & 6.03 & 4.73 & 4.62 & 1.04 & 1.41 \\
\hline \multicolumn{7}{|l|}{1999} \\
\hline Q1 & 5.46 & 5.71 & 4.56 & 4.57 & 0.90 & 1.14 \\
\hline Q2 & 5.24 & 5.50 & 4.53 & 4.62 & 0.72 & 0.88 \\
\hline Q3 & 5.06 & 5.33 & 4.86 & 5.12 & 0.20 & 0.21 \\
\hline Q4 & 5.32 & 5.58 & 5.50 & 5.51 & -0.18 & 0.07 \\
\hline \multicolumn{7}{|l|}{2000} \\
\hline Q1 & 5.47 & 5.67 & 5.56 & 5.73 & -0.09 & -0.06 \\
\hline Q2 & 5.44 & 5.74 & 6.06 & 6.26 & -0.62 & -0.52 \\
\hline Q3 & 5.45 & 5.76 & 6.20 & 6.33 & -0.75 & -0.57 \\
\hline Q4 & 5.35 & 5.64 & 6.15 & 6.16 & -0.80 & -0.51 \\
\hline \multicolumn{7}{|l|}{2001} \\
\hline Q1 & 4.59 & 4.80 & 4.88 & 4.74 & -0.29 & 0.07 \\
\hline Q2 & 3.77 & 3.91 & 3.73 & 3.68 & 0.04 & 0.23 \\
\hline Q3 & 3.60 & 3.74 & 3.01 & 3.00 & 0.59 & 0.74 \\
\hline Q4 & 2.85 & 3.00 & 1.70 & 1.72 & 1.15 & 1.28 \\
\hline \multicolumn{7}{|l|}{2002} \\
\hline Q1 & 2.61 & 2.76 & 1.40 & 1.52 & 1.20 & 1.24 \\
\hline Q2 & 2.46 & 2.62 & 1.45 & 1.59 & 1.01 & 1.03 \\
\hline Q3 & 2.12 & 2.26 & 1.37 & 1.40 & 0.75 & 0.86 \\
\hline Q4 & 1.71 & 1.75 & 1.08 & 1.10 & 0.63 & 0.65 \\
\hline \multicolumn{7}{|l|}{2003} \\
\hline Q1 & 1.44 & 1.49 & 0.86 & 0.90 & 0.58 & 0.60 \\
\hline Q2 & 1.51 & 1.58 & 0.78 & 0.80 & 0.73 & 0.78 \\
\hline Q3 & 1.50 & 1.57 & 0.66 & 0.69 & 0.83 & 0.88 \\
\hline
\end{tabular}

Source: Central Bank of Kuwait 
Table 25. Kuwait: Balance Sheet of the Investment Companies, 1997-2003

(In millions of Kuwaiti dinars)

\begin{tabular}{|c|c|c|c|c|c|c|c|}
\hline End of Period & 1997 & 1998 & 1999 & 2000 & 2001 & 2002 & $\frac{\text { June }}{2003}$ \\
\hline Assets & 2,788 & 3,458 & 3,256 & 3,518 & 3,418 & 3,492 & 3,882 \\
\hline Cash and balances with local banks & 176 & 259 & 208 & 154 & 168 & 134 & 136 \\
\hline Loans and discounts to residents & 275 & 354 & 349 & 346 & 437 & 451 & 499 \\
\hline Local investments & 807 & 891 & 588 & 421 & 415 & 519 & 700 \\
\hline Foreign assets & 1,364 & 1,686 & 1,868 & 2,249 & 2,321 & 2,285 & 2,422 \\
\hline Cash and balances with foreign banks & 173 & 142 & 134 & 104 & 147 & 62 & 99 \\
\hline Loans and discounts to nonresidents & 96 & 182 & 200 & 168 & 145 & 72 & 65 \\
\hline Foreign investments & 1,078 & 1,334 & 1,495 & 1,922 & 1,801 & 1,964 & 2,095 \\
\hline Other & 17 & 28 & 39 & 55 & 228 & 187 & 163 \\
\hline Other assets & 166 & 269 & 243 & 349 & 78 & 103 & 125 \\
\hline Liabilities & 2,788 & 3,459 & 3,256 & 3,518 & 3,418 & 3,492 & 3,882 \\
\hline Resources from residents & 560 & 622 & 394 & 298 & 454 & 503 & 581 \\
\hline From government & 94 & 85 & 7 & 7 & 7 & 0 & 1 \\
\hline From local banks & 226 & 296 & 208 & 240 & 393 & 417 & 492 \\
\hline From others & 240 & 241 & 179 & 51 & 54 & 86 & 88 \\
\hline Foreign liabilities & 711 & 1,057 & 1,124 & 1,400 & 1,425 & 1,389 & 1,560 \\
\hline Other liabilities & 387 & 426 & 428 & 471 & 305 & 375 & 434 \\
\hline Capital and reserves & 1,112 & 1,325 & 1,271 & 1,296 & 1,005 & 1,038 & 1,145 \\
\hline Other $1 /$ & 17 & 28 & 39 & 55 & 228 & 187 & 162 \\
\hline
\end{tabular}

Memorandum item:

Number of companies covered

28

34

37

36

25

26

26

Source: Central Bank of Kuwait.

1/ From 2001 onward, including only conventional companies. 
Table 26. Kuwait: Debt Collection Program - Repayments in 1995-2001

Repayment by Debtors Under the Law 41/93

\begin{tabular}{|c|c|c|c|c|}
\hline & \multicolumn{2}{|c|}{ Debtors } & \multicolumn{2}{|c|}{ Amounts Paid } \\
\hline & Number & Percent & KD Million & Percent \\
\hline \multicolumn{5}{|c|}{ First installment, 1995} \\
\hline Total & 2,850 & 100 & 449 & 100.00 \\
\hline Obligations met & 2,116 & 74 & 341 & 75.95 \\
\hline Default debtors & 734 & 26 & 108 & 24.05 \\
\hline \multicolumn{5}{|c|}{ First installment, 1996 1/ } \\
\hline Total due & 997 & 100.00 & 139 & 100.00 \\
\hline Obligations met & 437 & 43.83 & 104 & 74.82 \\
\hline Default debtors & 560 & 56.17 & 35 & 25.18 \\
\hline \multicolumn{5}{|c|}{ Second installment, 1997} \\
\hline Total due & 437 & 100.00 & 71 & 100.00 \\
\hline Obligations met & 295 & 67.51 & 56 & 78.87 \\
\hline Default debtors & 142 & 32.49 & 15 & 21.13 \\
\hline \multicolumn{5}{|c|}{ Third installment, 1998} \\
\hline Total due & 295 & 100.00 & 50 & 100.00 \\
\hline Obligations met & 249 & 84.41 & 46 & 92.00 \\
\hline Default debtors & 46 & 15.59 & 4 & 8.00 \\
\hline \multicolumn{5}{|c|}{ Fourth installment, 1999} \\
\hline Total due & 249 & 100.00 & 9 & 100.00 \\
\hline Obligations met & 73 & 29.32 & 5 & 55.56 \\
\hline Default debtors & 176 & 70.68 & 4 & 44.44 \\
\hline \multicolumn{5}{|c|}{ Fifth installment, 2000} \\
\hline Total due & 73 & 100.00 & 4 & 100.00 \\
\hline Obligations met & 10 & 13.70 & 3 & 75.00 \\
\hline Default debtors & 63 & 86.30 & 1 & 25.00 \\
\hline \multicolumn{5}{|c|}{ Sixth installment, $20012 /$} \\
\hline Total due & 10 & 100.00 & 1 & 100.00 \\
\hline Obligations met & 0 & 0.00 & 0 & 0.00 \\
\hline Default debtors & 10 & 100.00 & 1 & 100.00 \\
\hline \multirow{2}{*}{\multicolumn{5}{|c|}{$\begin{array}{l}\text { Status of clients subject to law } \\
\text { No. (41) for the year } 1993 \text { and } \\
\text { its amendments (2002) }\end{array}$}} \\
\hline & & & & \\
\hline Total due & 11,743 & 100.00 & 2,198 & 100.00 \\
\hline Obligations met & 9,942 & 84.66 & 1,759 & 80.03 \\
\hline Default debtors & 1,801 & 15.34 & 439 & 19.97 \\
\hline
\end{tabular}

Source: Central Bank of Kuwait (CBK).

1/ The data shown above concerning installments "1 to 3" have been updated due to change in Law 41/93 by Law 63/98.

2/ In accordance with Law 41/93 and its amendments, the sixth installment was due in June 2001.

This was the last installment and default debtors were referred by the CBK to the Public Prosecution. 
Table 27. Kuwait: Summary Balance of Payments, 1997-2002

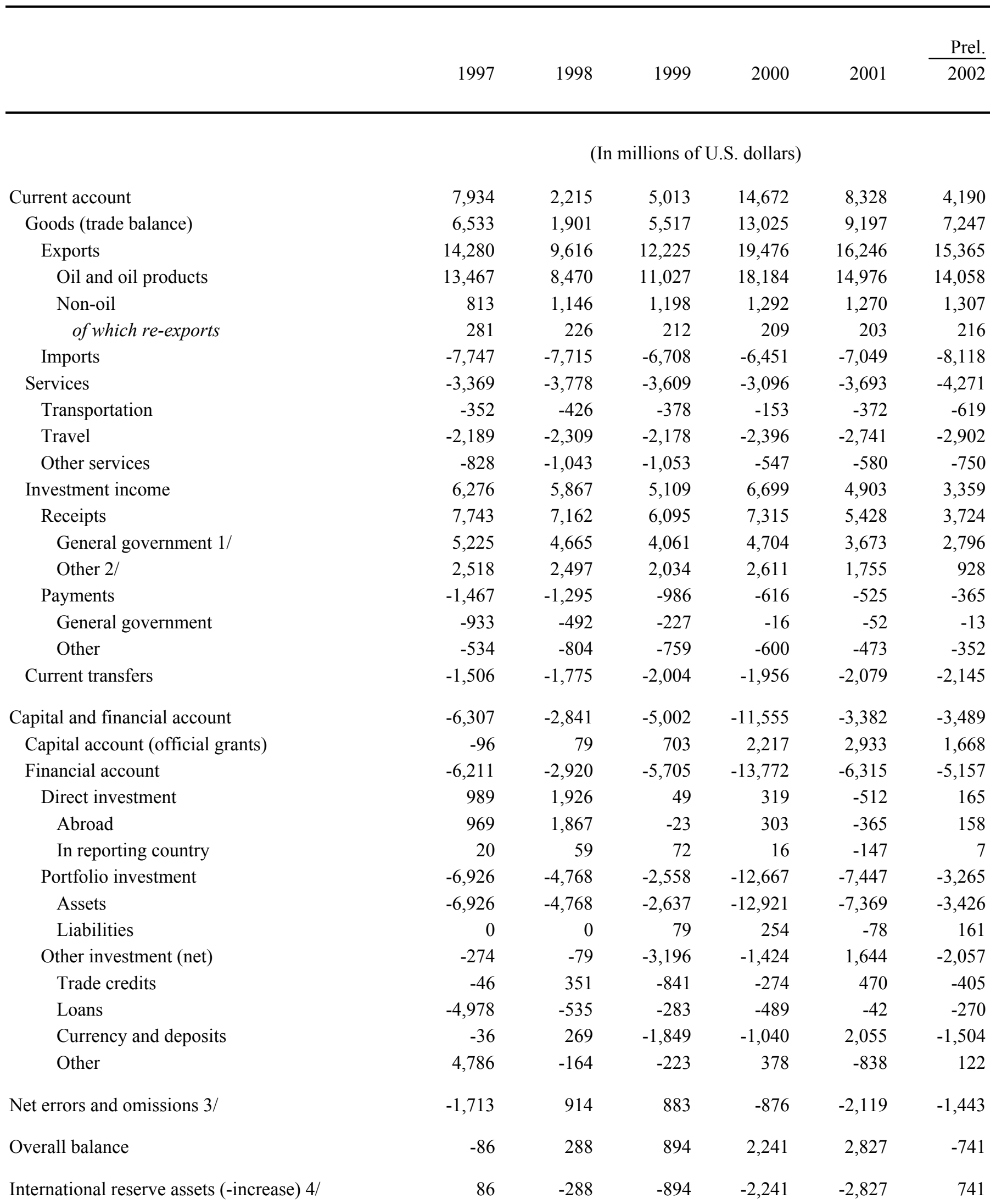


Table 27. Kuwait: Summary Balance of Payments, 1997-2002

$1997 \quad 1998 \quad 1999 \quad 2000 \quad 2001 \quad \frac{\text { Prel. }}{2002}$

Memorandum items:

$$
\text { (In percent of GDP) }
$$

\section{Current account}

Overall balance

International reserve assets

In months of imports of goods and services
26.1

$-0.3$

3,747

3.5
8.5

1.1

16.6

3.0

4,034

3.7

4,928

5.0

39.6

24.3

11.9

6.1

8.3

$-2.1$

7,170

9,997

9,256

7.4

9.7

Sources: Central Bank of Kuwait; and Fund staff estimates.

1/ Kuwait Investment Authority, Kuwait Petroleum Corporation, Kuwait Fund for Arab Economic Development, Public Institute for Social Security, Kuwait Airways Corporation, and Bank of Savings and Credit.

2/ Central Bank of Kuwait, local banks, investment, insurance, exchange companies, and nonfinancial private sector.

3/ Includes other unclassified private sector flows.

4/ As reported in the internationl investment position table. 
Table 28. Kuwait: Composition of Exports, 1997-2002

(In millions of U.S. dollars)

\begin{tabular}{|c|c|c|c|c|c|c|}
\hline & 1997 & 1998 & 1999 & 2000 & 2001 & 2002 \\
\hline Total f.o.b. & 14,280 & 9,616 & 12,225 & 19,476 & 16,246 & 15,363 \\
\hline \multicolumn{7}{|l|}{ Of which } \\
\hline Re-exports & 281 & 226 & 212 & 209 & 203 & 216 \\
\hline Oil and oil products & 13,466 & 8,471 & 11,027 & 18,184 & 14,976 & 14,057 \\
\hline Crude oil & 6,958 & 4,500 & 5,442 & 11,177 & 9,592 & 8,143 \\
\hline Refined products & 5,735 & 3,542 & 4,977 & 6,132 & 4,589 & 5,273 \\
\hline \multicolumn{7}{|l|}{ Of which } \\
\hline Bunker oil & 194 & 111 & 0 & 0 & 0 & 0 \\
\hline LPG & 772 & 428 & 608 & 875 & 795 & 642 \\
\hline Non-oil & 756 & 1,082 & 1,138 & 1,252 & 1,236 & 1,294 \\
\hline Ethylene products & 25 & 435 & 530 & 640 & 621 & 628 \\
\hline Fertilizers & 100 & 65 & 57 & 64 & 70 & 51 \\
\hline Road vehicle & 124 & 108 & 57 & 53 & 67 & 68 \\
\hline Nonmetallic minerals & 35 & 30 & 34 & 34 & 35 & 40 \\
\hline Electrical manufactures & 32 & 33 & 29 & 28 & 32 & 33 \\
\hline Paper products & 25 & 25 & 25 & 25 & 30 & 33 \\
\hline Metallic cores and scrap & 33 & 32 & 34 & 37 & 33 & 40 \\
\hline Metal products & 37 & 37 & 41 & 30 & 29 & 27 \\
\hline Other & 344 & 318 & 331 & 341 & 319 & 375 \\
\hline \multicolumn{7}{|l|}{ Adjustments for BOP } \\
\hline Unrecorded exports $1 /$ & 58 & 63 & 60 & 40 & 34 & 12 \\
\hline
\end{tabular}

Sources: Central Bank of Kuwait; Ministry of Oil; Central Statistical Office.

1/ Exports not reflected by the customs returns. 
Table 29. Kuwait: Composition of Imports, 1997-2002

(In millions of U.S. dollars)

\begin{tabular}{|c|c|c|c|c|c|c|}
\hline & 1997 & 1998 & 1999 & 2000 & 2001 & 2002 \\
\hline Total, f.o.b. & 7,747 & 7,713 & 6,709 & 6,451 & 7,050 & 8,116 \\
\hline By SITC category (c.i.f.) & 8,246 & 8,616 & 7,616 & 7,156 & 7,872 & 9,001 \\
\hline Food and live animals & 1,129 & 1,169 & 1,135 & 1,138 & 1,158 & 1,260 \\
\hline Beverages and tobacco & 87 & 87 & 100 & 68 & 65 & 82 \\
\hline Crude materials & 108 & 154 & 145 & 145 & 173 & 211 \\
\hline Mineral fuels & 44 & 38 & 49 & 43 & 39 & 46 \\
\hline Other oils and fats & 43 & 42 & 43 & 41 & 39 & 39 \\
\hline Chemicals & 690 & 668 & 660 & 678 & 724 & 783 \\
\hline Manufactured materials & 1,658 & 1,577 & 1,243 & 1,288 & 1,475 & 1,681 \\
\hline Machinery and equipment & 3,180 & 3,550 & 3,024 & 2,673 & 2,975 & 3,583 \\
\hline Miscellaneous manufactures & 1,163 & 1,180 & 1,107 & 992 & 1,139 & 1,244 \\
\hline Other commodities & 145 & 153 & 111 & 91 & 85 & 72 \\
\hline By economic use (c.i.f.) & 8,246 & 8,616 & 7,616 & 7,156 & 7,872 & 9,001 \\
\hline Capital goods & 1,522 & 1,734 & 1,367 & 1,093 & 1,187 & 1,382 \\
\hline Intermediate goods & 3,026 & 3,032 & 2,618 & 2,625 & 2,848 & 3,405 \\
\hline Consumer goods & 3,551 & 3,694 & 3,516 & 3,345 & 3,748 & 4,139 \\
\hline Other, unspecified & 147 & 156 & 115 & 94 & 88 & 76 \\
\hline Adjustments for BOP & -500 & -903 & -908 & -705 & $-2,587$ & $-2,911$ \\
\hline Unrecorded imports $1 /$ & 737 & 390 & 235 & 368 & $-1,406$ & $-1,563$ \\
\hline Freight and insurance payments & $-1,237$ & $-1,292$ & $-1,142$ & $-1,073$ & $-1,181$ & $-1,349$ \\
\hline
\end{tabular}

Source: Ministry of Planning, Central Statistical Office.

1/ Imports not reflected by the customs returns. 
Table 30. Kuwait: External Services, Investment Income, and Current Transfers, 1997-2002

(In millions of U.S. dollars)

\begin{tabular}{|c|c|c|c|c|c|c|}
\hline & 1997 & 1998 & 1999 & 2000 & 2001 & 2002 \\
\hline Receipts & 9,583 & 9,023 & 7,753 & 9,221 & 7,144 & 5,422 \\
\hline Transport 1/ & 1,220 & 1,198 & 1,120 & 1,382 & 1,204 & 1,128 \\
\hline Insurance 1/ & 69 & 72 & 66 & 72 & 72 & 109 \\
\hline Travel & 188 & 207 & 92 & 98 & 104 & 118 \\
\hline Government, n.i.e. & 254 & 266 & 263 & 251 & 264 & 276 \\
\hline Other services & 30 & 20 & 20 & 20 & 20 & 16 \\
\hline Investment income & 7,743 & 7,162 & 6,094 & 7,315 & 5,428 & 3,724 \\
\hline Government 2/ & 5,386 & 4,833 & 4,061 & 4,951 & 3,967 & 3,020 \\
\hline Private & 2,357 & 2,329 & 2,034 & 2,363 & 1,461 & 704 \\
\hline Financial institutions 3/ & 794 & 945 & 628 & 724 & 470 & 273 \\
\hline Other & 1,563 & 1,385 & 1,406 & 1,640 & 992 & 431 \\
\hline Government transfer receipts & 79 & 98 & 99 & 85 & 52 & 49 \\
\hline Payments & $-8,182$ & $-8,711$ & $-8,259$ & $-7,575$ & $-8,012$ & $-8,478$ \\
\hline Transport & $-1,572$ & $-1,624$ & $-1,498$ & $-1,535$ & $-1,576$ & $-1,747$ \\
\hline Insurance & -66 & -69 & -62 & -59 & -62 & -72 \\
\hline Travel & $-2,377$ & $-2,516$ & $-2,270$ & $-2,494$ & $-2,845$ & $-3,020$ \\
\hline Government, n.i.e. & $-1,075$ & $-1,299$ & $-1,304$ & -805 & -835 & $-1,040$ \\
\hline Other services & -40 & -33 & -36 & -26 & -39 & -39 \\
\hline Investment income & $-1,467$ & $-1,296$ & -986 & -616 & -525 & -365 \\
\hline Government & -933 & -492 & -227 & -16 & -52 & -13 \\
\hline Private & -534 & -804 & -759 & -600 & -473 & -352 \\
\hline Financial institutions & -392 & -650 & -453 & -447 & -333 & -217 \\
\hline Other & -142 & -154 & -306 & -153 & -140 & -135 \\
\hline Government transfer payment & -178 & -217 & -171 & -225 & -258 & -174 \\
\hline Private transfer payments 4/ & $-1,408$ & $-1,657$ & $-1,932$ & $-1,816$ & $-1,873$ & $-2,020$ \\
\hline Total (net) & 1,401 & 312 & -506 & 1,646 & -868 & $-3,056$ \\
\hline Insurance & 3 & 3 & 3 & 13 & 10 & 36 \\
\hline Transport & -353 & -427 & -378 & -153 & -372 & -618 \\
\hline Travel & $-2,189$ & $-2,310$ & $-2,178$ & $-2,396$ & $-2,740$ & $-2,902$ \\
\hline Government, n.i.e. & -821 & $-1,033$ & $-1,041$ & -554 & -571 & -763 \\
\hline Other services & -10 & -13 & -16 & -7 & -20 & -23 \\
\hline Investment income & 6,276 & 5,866 & 5,109 & 6,698 & 4,903 & 3,359 \\
\hline Government & 4,453 & 4,341 & 3,985 & 4,935 & 3,915 & 3,007 \\
\hline Private & 1,823 & 1,526 & 1,124 & 1,763 & 988 & 352 \\
\hline Financial institutions & 402 & 295 & 174 & 277 & 137 & 56 \\
\hline Other & 1,421 & 1,230 & 949 & 1,486 & 851 & 296 \\
\hline Government transfer & -99 & -118 & -72 & -140 & -206 & -125 \\
\hline Private transfer & $-1,408$ & $-1,657$ & $-1,932$ & $-1,816$ & $-1,873$ & $-2,020$ \\
\hline
\end{tabular}

Source: Central Bank of Kuwait.

1/ Available data are prepared according to BPM5 which separate transport from insurance.

2/ Income from external assets managed by Central Bank of Kuwait (CBK), Kuwait Investment Authority (KIA), Kuwait Petroleum Corporation (KPC), and Kuwait Airways Corporation (KAC).

3 / Income from external assets of local banks and investment companies.

4/ Includes remittances of long-term expatriate workers. 
Table 31. Kuwait: Capital and Financial Account, 1998-2002

(In millions of U.S. dollars)

\begin{tabular}{|c|c|c|c|c|c|}
\hline & 1998 & 1999 & 2000 & 2001 & 2002 \\
\hline Capital and Financial Account & $-2,841$ & $-5,004$ & $-11,555$ & $-3,383$ & $-3,487$ \\
\hline Capital account & 79 & 703 & 2,217 & 2,933 & 1,668 \\
\hline General Government & -210 & -13 & 69 & 1,207 & 283 \\
\hline Other sectors & 289 & 716 & 2,148 & 1,726 & 1,385 \\
\hline Financial Account & $-2,920$ & $-5,707$ & $-13,772$ & $-6,316$ & $-5,155$ \\
\hline Direct investment & 1,926 & 49 & 319 & -512 & 164 \\
\hline Direct investment abroad & 1,867 & -23 & 303 & -365 & 158 \\
\hline General government & 1,860 & -36 & 297 & -362 & 171 \\
\hline Other sectors & 7 & 13 & 7 & -3 & -13 \\
\hline Foreign direct investment in Kuwait & 59 & 72 & 16 & -147 & 7 \\
\hline Portfolio investment (net) & $-4,767$ & $-2,559$ & $-12,667$ & $-7,448$ & $-3,263$ \\
\hline \multicolumn{6}{|l|}{ Assets } \\
\hline General government & $-4,045$ & $-2,300$ & $-12,005$ & $-7,882$ & $-1,925$ \\
\hline Local banks & 154 & 168 & 411 & -434 & -763 \\
\hline Investment companies & -876 & -506 & $-1,327$ & 946 & -737 \\
\hline Liabilities & 0 & 79 & 254 & -78 & 161 \\
\hline Local banks & 0 & 105 & 254 & -78 & 161 \\
\hline Investment companies & 0 & -26 & 0 & 0 & 0 \\
\hline Other investment (net) & -79 & $-3,197$ & $-1,424$ & 1,644 & $-2,056$ \\
\hline Assets & 646 & $-3,512$ & $-1,108$ & 506 & $-3,750$ \\
\hline Trade credit & 538 & -736 & -130 & 470 & -405 \\
\hline Loans & -289 & -463 & -313 & -13 & -86 \\
\hline General government & -39 & -493 & -535 & -476 & -125 \\
\hline Local banks & 33 & 89 & 114 & 388 & -197 \\
\hline Investment companies & -282 & -59 & 108 & 75 & 237 \\
\hline Currency and deposits & 715 & $-2,152$ & -711 & 760 & $-2,872$ \\
\hline General government & -236 & $-1,945$ & 329 & 1,034 & $-2,731$ \\
\hline Local banks & 902 & -309 & $-1,115$ & -130 & -408 \\
\hline Other sectors & 49 & 102 & 75 & -144 & 266 \\
\hline Other & -318 & -161 & 46 & -711 & -388 \\
\hline General government & -272 & -115 & 52 & -127 & -618 \\
\hline Local banks & -7 & 59 & -3 & -16 & 7 \\
\hline Other sectors & -39 & -105 & -3 & -568 & 224 \\
\hline
\end{tabular}


Table 31. Kuwait: Capital and Financial Account, 1998-2002

(In millions of U.S. dollars)

\begin{tabular}{lrrrrr}
\hline & 1998 & 1999 & 2000 & 2001 & 2002 \\
& & & & & \\
\hline & & & & & \\
Liabilities & -725 & 315 & -316 & 1,139 & 1,694 \\
Trade credit & -187 & -105 & -143 & 0 & 0 \\
Loans & -246 & 181 & -176 & -29 & -184 \\
General government & $-1,125$ & -36 & -851 & -33 & -46 \\
Other sectors & 879 & 217 & 675 & 3 & -138 \\
Currency and deposits & -446 & 302 & -329 & 1,295 & 1,369 \\
Other & & & & & 0 \\
General government & -3 & 26 & 156 & 0 & -13 \\
Local banks & -10 & 0 & 33 & 7 & 49 \\
Other sectors & 167 & -89 & 137 & -134 & \\
& & & & & \\
\end{tabular}

Source: Central Bank of Kuwait. 
Table 32. Kuwait: Reserves and Net Foreign Assets, 1997-2002

(In millions of U.S. dollars)

\begin{tabular}{|c|c|c|c|c|c|c|}
\hline & 1997 & 1998 & 1999 & 2000 & 2001 & 2002 \\
\hline Central bank net foreign assets & 3,747 & 4,034 & 4,926 & 7,170 & 9,997 & 9,256 \\
\hline International gross reserve assets & 3,765 & 4,041 & 4,934 & 7,183 & 10,008 & 9,296 \\
\hline Central bank foreign assets & 3,415 & 3,589 & 4,347 & 6,571 & 9,284 & 8,451 \\
\hline Gold 1/ & 104 & 105 & 104 & 104 & 103 & 106 \\
\hline Foreign exchange & 3,311 & 3,484 & 4,243 & 6,467 & 9,181 & 8,345 \\
\hline SDRs 2/ & 103 & 112 & 73 & 93 & 109 & 126 \\
\hline IMF reserve position & 247 & 340 & 514 & 519 & 615 & 719 \\
\hline Central bank foreign liabilities & 18 & 7 & 8 & 13 & 12 & 40 \\
\hline Local banks' net foreign assets $3 /$ & 2,951 & 2,308 & 1,901 & 2,530 & 1,484 & 1,381 \\
\hline Foreign assets & 6,981 & 5,866 & 5,869 & 6,440 & 6,594 & 8,160 \\
\hline Foreign liabilities & 4,030 & 3,558 & 3,968 & 3,910 & 5,110 & 6,779 \\
\hline Net foreign assets of the banking system & 6,698 & 6,343 & 6,827 & 9,700 & 11,481 & 10,637 \\
\hline \multicolumn{7}{|l|}{ Net foreign assets of nonbank financial institutions, } \\
\hline including insurance companies $4 /$ & 1,472 & 2,067 & 2,496 & 1,810 & 1,778 & 1,888 \\
\hline Foreign assets & 4,529 & 5,691 & 6,272 & 7,413 & 7,068 & 7,220 \\
\hline Foreign liabilities & 3,057 & 3,624 & 3,777 & 5,603 & 5,290 & 5,332 \\
\hline Net foreign assets of the financial sector & 8,170 & 8,409 & 9,323 & 11,510 & 13,259 & 12,525 \\
\hline \multicolumn{7}{|l|}{ Memorandum items: } \\
\hline Central bank foreign exchange at central bank valuation $5 /$ & 3,306 & 3,484 & 4,243 & 6,473 & 9,187 & $\ldots$ \\
\hline Central bank gross foreign assets $5 /$ & 3,428 & 3,552 & 4,344 & 6,547 & 9,308 & ... \\
\hline Central bank foreign exchange $6 /$ & 3,323 & 3,448 & 4,240 & 6,444 & 9,205 & ... \\
\hline \multicolumn{7}{|l|}{ Net foreign assets of nonbank financial institutions, } \\
\hline excluding insurance companies & 2,116 & 2,021 & 2,349 & 2,629 & 2,170 & ... \\
\hline Foreign assets & 4,497 & 5,586 & 6,089 & 7,257 & 6,807 & ... \\
\hline Foreign liabilities & 2,381 & 3,564 & 3,740 & 4,628 & 4,636 & $\ldots$ \\
\hline
\end{tabular}

Source: Central Bank of Kuwait.

1/ At national valuation of KD 12.5 per fine ounce.

2/ Carried as assets of the Ministry of Finance.

3/ Commercial banks, specialized banks, and Kuwait Finance House.

4/ Investment companies, exchange companies, insurance companies, and starting in 2000 Equate company.

5/ At period average exchange rate.

6/ At central bank evaluation as opposed to IFS valuation above and at period average exchange rate. 
Table 33. Kuwait: Aggregate Banking Soundness Indicators, 1997-2003

(End of period unless otherwise indicated)

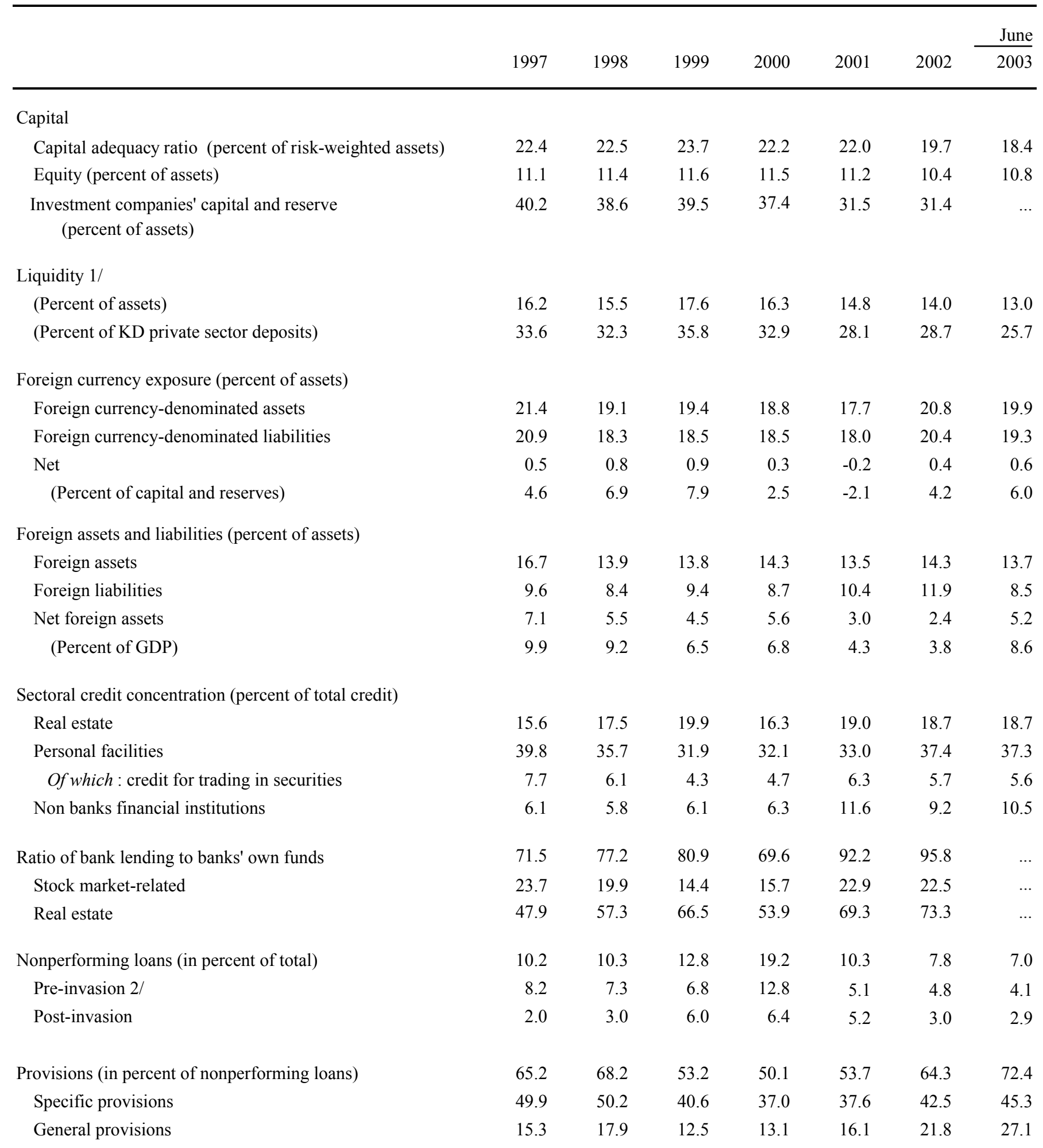


Table 33. Kuwait: Aggregate Banking Soundness Indicators, 1997-2003

(End of period unless otherwise indicated)

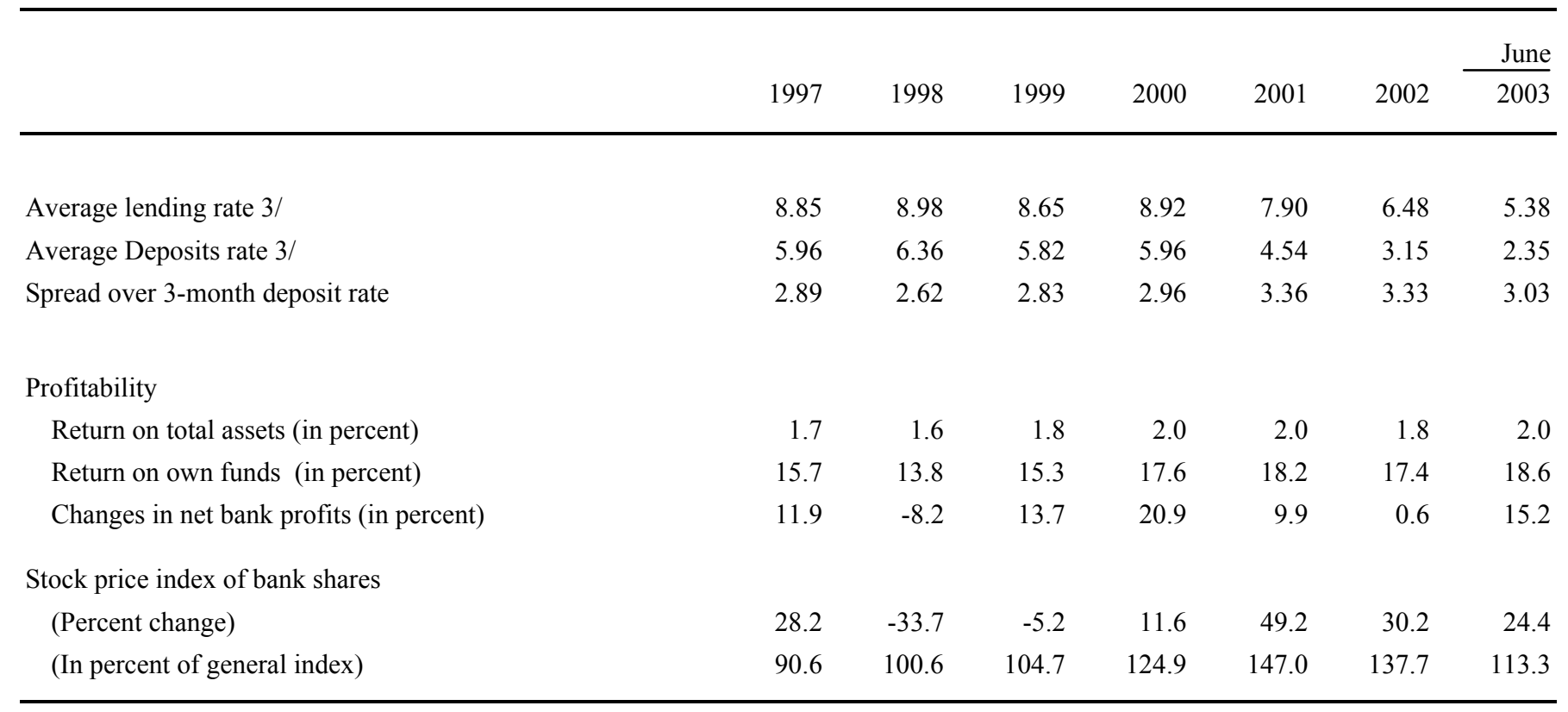

Sources: Central Bank of Kuwait; rating agencies; Global Finance House; and Fund staff estimates.

1/ Cash, central bank current deposits, and treasury bills and bonds.

2/ Guaranteed by government.

3/ For local banks. 
Table 34. Kuwait: Selected Stock Market Indicators, 1997-2003

\begin{tabular}{|c|c|c|c|c|c|c|c|}
\hline & 1997 & 1998 & 1999 & 2000 & 2001 & 2002 & $\frac{\text { Sept. }}{2003}$ \\
\hline Market index $(1993 / 12=1000) 1 /$ & 2,652 & 1,583 & 1,442 & 1,348 & 1,709 & 2,375 & 4,510 \\
\hline $\begin{array}{l}\text { Value of shares traded } \\
\text { (In millions of KD) }\end{array}$ & 10,487 & 3,341 & 1,841 & 1,332 & 3,581 & 6,680 & 12,412 \\
\hline $\begin{array}{l}\text { Number of shares traded } \\
\text { (In millions) }\end{array}$ & 33,988 & 13,917 & 9,495 & 6,921 & 16,300 & 27,837 & 38,650 \\
\hline $\begin{array}{l}\text { Number of transactions } \\
\text { (In thousands) }\end{array}$ & 588 & 350 & 212 & 159 & 354 & 521 & 822 \\
\hline $\begin{array}{l}\text { Market capitalization } \\
\text { (In millions of KD end-period) }\end{array}$ & 9,083 & 5,802 & 6,184 & 6,378 & 8,300 & 10,541 & 16,537 \\
\hline Memorandum items: & & & & & & & \\
\hline Number of listed companies & 74 & 78 & 85 & 86 & 88 & 95 & 103 \\
\hline Market capitalization/GDP & 100.3 & 75.8 & 69.6 & 56.2 & 79.1 & 98.2 & 154.0 \\
\hline Turnover $2 /$ & 115.5 & 57.6 & 29.8 & 20.9 & 43.1 & 63.4 & 75.1 \\
\hline $\begin{array}{l}\text { Profits of listed companies } \\
\text { (percentage change) }\end{array}$ & 190.4 & -43.9 & 27.1 & 9.3 & 26.5 & 14.1 & $\ldots$ \\
\hline Price/earnings ratio & 14.5 & 13.9 & 13.9 & 12.7 & 14.4 & 22.5 & $\ldots$ \\
\hline
\end{tabular}

Source: Central Bank of Kuwait.

1/ End-December 1993.

2/ Value of shares traded divided by market capitalization. 
Table 35. Kuwait: External Debt, 1997-2002

(In millions of U.S. dollars)

\begin{tabular}{|c|c|c|c|c|c|c|}
\hline & 1997 & 1998 & 1999 & 2000 & 2001 & 2002 \\
\hline Total external debt outstanding & 4,848 & 9,938 & 10,057 & 9,955 & 11,094 & 12,940 \\
\hline Total Interest repayments & 1,462 & 1,310 & 986 & 618 & 524 & 370 \\
\hline General government external debt 1 / & 2,726 & 1,419 & 1,289 & 442 & 410 & 827 \\
\hline Interest repayments & 930 & 497 & 227 & 16 & 52 & 13 \\
\hline Private external debt $2 /$ & 2,122 & 8,519 & 8,768 & 9,513 & 10,685 & 12,112 \\
\hline Interest repayments $3 /$ & 532 & 812 & 759 & 602 & 472 & 357 \\
\hline
\end{tabular}

Source: Central Bank of Kuwait.

1/ Includes year-end stocks of loans, repos and other credit facilities as defined in the BPM5.

2/ Local banks, investment, exchange, insurance companies, and Equate project.

3/ Partially estimated on the basis of LIBOR plus 0.5 percent and the average stock of the previous and current year, as data on other nonfinancial private sector debt are incomplete. 